ISSN 1996-1944

www.mdpi.com/journal/materials

Review

\title{
Hydrothermal Synthesis of Metal Oxide Nanoparticles in Supercritical Water
}

\section{Hiromichi Hayashi ${ }^{1, *}$ and Yukiya Hakuta ${ }^{2}$}

1 Research Center for Compact Chemical System, National Institute of Advanced Industrial Science and Technology, Nigatake-4-2-1, Miyagino-ku, Sendai, 983-8551, Japan

2 Nanosystem Research Institute, National Institute of Advanced Industrial Science and Technology, Azuma-1-1-1, Tsukuba, Ibaragi, 305-8565, Japan; E-Mail: y-hakuta@aist.go.jp

* Author to whom correspondence should be addressed; E-Mail: h-hayashi@aist.go.jp; Tel.: +81-22-237-2029; Fax: +81-22-237-5215.

Received: 2 June 2010; in revised form: 17 June 2010 / Accepted: 21 June 2010 /

Published: 25 June 2010

\begin{abstract}
This paper summarizes specific features of supercritical hydrothermal synthesis of metal oxide particles. Supercritical water allows control of the crystal phase, morphology, and particle size since the solvent's properties, such as density of water, can be varied with temperature and pressure, both of which can affect the supersaturation and nucleation. In this review, we describe the advantages of fine particle formation using supercritical water and describe which future tasks need to be solved.
\end{abstract}

Keywords: supercritical water; particle formation; nano-particle; micronization; hydrothermal synthesis

\section{Introduction}

Hydrothermal synthesis is generally defined as crystal synthesis or crystal growth under high temperature and high pressure water conditions from substances which are insoluble in ordinary temperature and pressure $\left(<100{ }^{\circ} \mathrm{C},<1 \mathrm{~atm}\right)$. Since ionic product $\left(\mathrm{K}_{\mathrm{w}}\right)$ has a maximum value of around $250-300{ }^{\circ} \mathrm{C}$, hydrothermal synthesis is usually carried out below $300{ }^{\circ} \mathrm{C}$. The critical temperature and pressure of water are $374{ }^{\circ} \mathrm{C}$ and $22.1 \mathrm{MPa}$, respectively. The solvent properties for many compounds, such as dielectric constant and solubility, change dramatically under supercritical 
conditions. The density of water and dielectric constant are shown in Figure 1 as a function of temperature and pressure. The dielectric constant of water is 78 at room temperature, where polar inorganic salts can be soluble in water. The dielectric constant of water decreases with increasing temperature and decreasing pressure. The dielectric constant is below 10 under supercritical conditions; the contribution of the dielectric constant to the reaction rates becomes remarkable based on the electrostatic theory. Thus, supercritical water gives a favorable reaction field for particle formation, owing to the enhancement of the reaction rate and large supersaturation based on the nucleation theory, due to lowering the solubility.

Figure 1. (a) Density of water as a function of temperature and pressure. (b) Dielectric constant as a function of temperature and pressure.
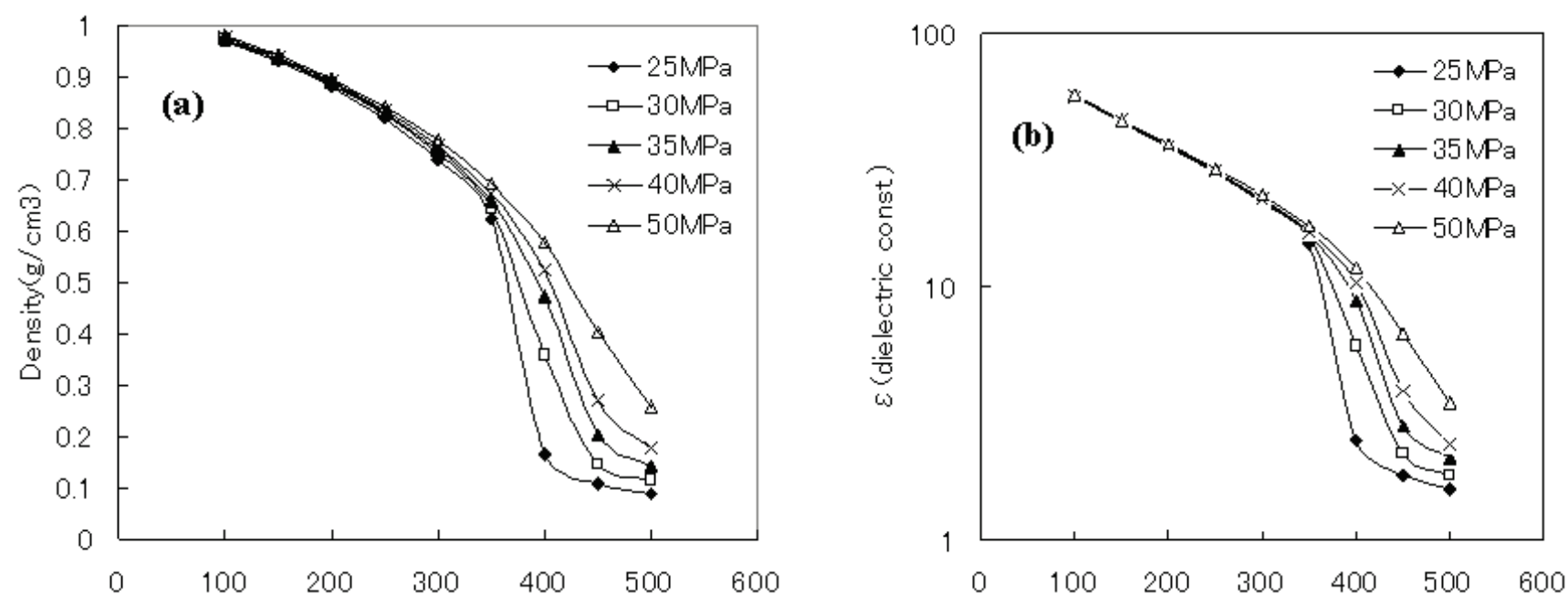

The formation mechanism of metal oxide particles from metal nitrate solution is as follows: First, hydrated metal ions are hydrolyzed to metal hydroxide. Then, metal hydroxides proceed to precipitate as metal oxides through dehydration [1,2].

$$
\begin{aligned}
& \mathrm{M}\left(\mathrm{NO}_{3}\right)_{\mathrm{x}}+\mathrm{xH}_{2} \mathrm{O}=\mathrm{M}(\mathrm{OH})_{\mathrm{x}}+\mathrm{xHNO}_{3} \\
& \mathrm{M}(\mathrm{OH})_{\mathrm{x}}=\mathrm{MO}_{\mathrm{x} / 2}+\mathrm{x} / 2 \mathrm{H}_{2} \mathrm{O}
\end{aligned}
$$

Hydrolysis is regarded as an electrostatic reaction between metal ions and hydroxyl ions. The electrostatic contribution to the reaction rate can be expressed as Equation (3) using ionic species between $\mathrm{A}^{\mathrm{zA}}$ and $\mathrm{B}^{\mathrm{zB}}$ in aqueous solution via activated complex $\left\{\mathrm{AB}^{\mathrm{ZAB}}\right\}^{\#}$.

$$
\begin{aligned}
& \mathrm{A}^{\mathrm{zA}}+\mathrm{B}^{\mathrm{zB}}=\left\{\mathrm{AB}^{\mathrm{zAB}}\right\}^{\#} \rightarrow \mathrm{AB}^{\mathrm{zAB}} \\
& \ln k=\ln k_{0}+\frac{N e^{2}}{2 R T}\left(\frac{z_{A B}^{2}}{r_{A B}}-\left\{\frac{z_{A B}^{2}}{r_{A B}}\right\} \#\right)\left(\frac{1}{\varepsilon}-\frac{1}{\varepsilon_{0}}\right)
\end{aligned}
$$

Where $\mathrm{z}$ and $\mathrm{r}$ represent charge and radius of ionic species, the first term is the reaction rate in the solvent with dielectric constant $\varepsilon_{0}$ and the second term expresses the solvent effect of the solvent with dielectric constant $\varepsilon$. 
Hydrothermal synthesis in supercritical water has advantages for synthesis of multi metal oxide compounds because the reaction rate is enhanced more than $10^{3}$ times that under the conventional hydrothermal conditions owing to the low dielectric constant $(<10)$ as well as products with high crystallinity [1-3]. The particle size of metal oxide depends on the hydrolysis rate and solubility of the metal oxide. To achieve the control of the solvent field during nucleation and crystallization of particles, hydrothermal conditions of temperature and pressure can be varied in subcritical and supercritical water. Hydrothermal methods for preparing fine metal oxide particles in subcritical and supercritical water have been developed using batch reaction [4-16,32-42] and flow reaction systems [17-28,43-81]. The production of various metal oxide particles such as $\mathrm{TiO}_{2}$ [4,27], $\mathrm{K}_{2} \mathrm{Ti}_{6} \mathrm{O}_{13}$ [5,17,18], $\mathrm{K}_{4} \mathrm{Nb}_{6} \mathrm{O}_{17}$ [6], $\mathrm{KNbO}_{3}$ [7], $\mathrm{KTiNbO}_{3}$ [8-10], $\mathrm{KTaO}_{3}$ [11], $\mathrm{Zn}_{2} \mathrm{SiO}_{4}: \mathrm{Mn}$ [12-16], $\mathrm{ZrO}_{2}$ [19,27,67], $\mathrm{AlOOH}[20], \mathrm{Al}_{2} \mathrm{O}_{3}$ [21,69], $\mathrm{Ba}(\mathrm{Sr}) \mathrm{Ti}(\mathrm{Zr}) \mathrm{O}_{3}$ [22-24,51-54,72,73], $\mathrm{Ca}_{0.8} \mathrm{Sr}_{0.2} \mathrm{Ti}_{1-\mathrm{x}} \mathrm{FeO}_{3-}$ [25], YSZ [26], (Fe,In) $)_{2} \mathrm{O}_{3}(\mathrm{ITO})$ [32,57], $\mathrm{LiFePO}_{4}$ [33,71], (Ce,Zr)O $\mathrm{O}_{2}$ [34,39,77,80,81], $\mathrm{YVO}_{4}$ [35], $(\mathrm{Co}, \mathrm{Cu}, \mathrm{Ni})(\mathrm{Fe}, \mathrm{Co})_{2} \mathrm{O}_{4}[36,45,74,78], \mathrm{Fe}_{2} \mathrm{O}_{3}$ [37,70], YAG [38,46,58,59], ErOOH [40], $\mathrm{Mg}_{3.5} \mathrm{H}_{2}\left(\mathrm{PO}_{4}\right)_{3}$ [41], $\mathrm{CuAlO}_{2}$ [42], $\mathrm{ZnO}$ [47-49,79], $\mathrm{LiMn}_{2} \mathrm{O}_{4}$ [55], $\mathrm{La}_{\mathrm{x}} \mathrm{Ni}_{\mathrm{y}} \mathrm{O}_{3}$ [60,76], $\mathrm{SnO}_{2}$ [68], $(\mathrm{Ca}, \mathrm{Mg})\left(\mathrm{PO}_{4}\right)_{3}$ [75] has been demonstrated by hydrothermal batch and flow reaction systems. Supercritical water has specific properties inducing low solubility of inorganics but high solubility of organics, due to its low dielectric constant, which makes it suitable for the synthesis of hybrid nanoparticles. Adschiri et al. have developed an in situ surface modification technique of nanoparticles with organics using supercritical flow reaction system [62-66]. Readers interested in the topic of the supercritical flow reaction system for hybrid nanoparticles should consult the original review articles $[1,2]$. In this review, we focus on advantages of supercritical hydrothermal methods based on our results in batch and flow reaction systems. In the case of the batch reaction system, the advantage for synthesis of metal oxides in supercritical water is reducing alkaline concentration for the crystal growth. We have demonstrated hydrothermal synthesis of potassium niobate and potassium tantalate powders with various subcritical and supercritical conditions under low KOH concentration (0.1-0.5 M). Single phase of $\mathrm{KNbO}_{3}$ and $\mathrm{KTaO}_{3}$ can be achieved by hydrothermal synthesis in supercritical water even under low $\mathrm{KOH}$ concentration $[6,7,11]$. In the case of the flow reaction system, the density of water can be varied with the temperature and pressure under supercritical conditions, whereas synthetic conditions are rarely varied with wide range of density of water in supercritical water. The crystallite phase can be controlled with the density of water. $\gamma-\mathrm{Al}_{2} \mathrm{O}_{3}$ nanoparticles were obtained at $410{ }^{\circ} \mathrm{C}$ or higher where the density of water is $0.25 \mathrm{~g} / \mathrm{cm}^{3}$ or lower [21]. Tetragonal barium titanate $\left(\mathrm{BaTiO}_{3}\right)$ can be obtained when the density of water was smaller than $0.5 \mathrm{gcm}^{-3}$ [22-24].

\section{Hydrothermal Synthesis of Metal Oxide}

\subsection{Batch Reaction System}

The starting materials, synthetic conditions, product phases and particle sizes of the materials obtained by hydrothermal batch reaction are described in Table 1 and scanning electron micrographs (SEM) of representative particles are shown in Figure 2. The advantages of hydrothermal synthesis under supercritical water conditions were demonstrated to prepare highly active photocatalysts. Different preparation methods have important effects on the resulting microstructure and physical 
properties of the materials. $\mathrm{TiO}_{2}$ particles synthesized hydrothermally under supercritical conditions have high crystallinity and large surface areas which are responsible for good photocatalytic performance [4]. Potassium hexatitanate (KTO) synthesized hydrothermally under sub- and supercritical water conditions produce thermally stable long, felt-like, thin fibers (Figure 2(a)) of large surface area as compared to short, thick fibers in the solid-state method. The growth of these long fibrous crystals is more pronounced along the b-axis (020). The photocatalytic activities for the water decomposition over $\mathrm{RuO}_{2}$ /hydrothermally synthesized $\mathrm{KTO}$ are remarkably higher than those over $\mathrm{RuO}_{2}$ loaded solid-state synthesized KTO photocatalysts. In particular, KTO hydrothermally synthesized under super-critical conditions exhibits higher activity than KTO synthesized under subcritical conditions. The crystallinity of the KTO seems beneficial to a certain extent for high photocatalytic performance. The KTO with optimum crystallite size of about $20 \mathrm{~nm}$ and surface area of $38 \mathrm{~m}^{2} / \mathrm{g}$ seems to have highest activity [5].

Figure 2. Scanning electron microscopy (SEM) images of metal oxide particles synthesized by supercritical hydrothermal batchwise system. (a) $\mathrm{K}_{2} \mathrm{Ti}_{6} \mathrm{O}_{13}$, (b) $\mathrm{Zn}_{2} \mathrm{SiO}_{4}: \mathrm{Mn}$, (c) $\mathrm{KNbO}_{3}$, (d) $\mathrm{KTaO}_{3}$.
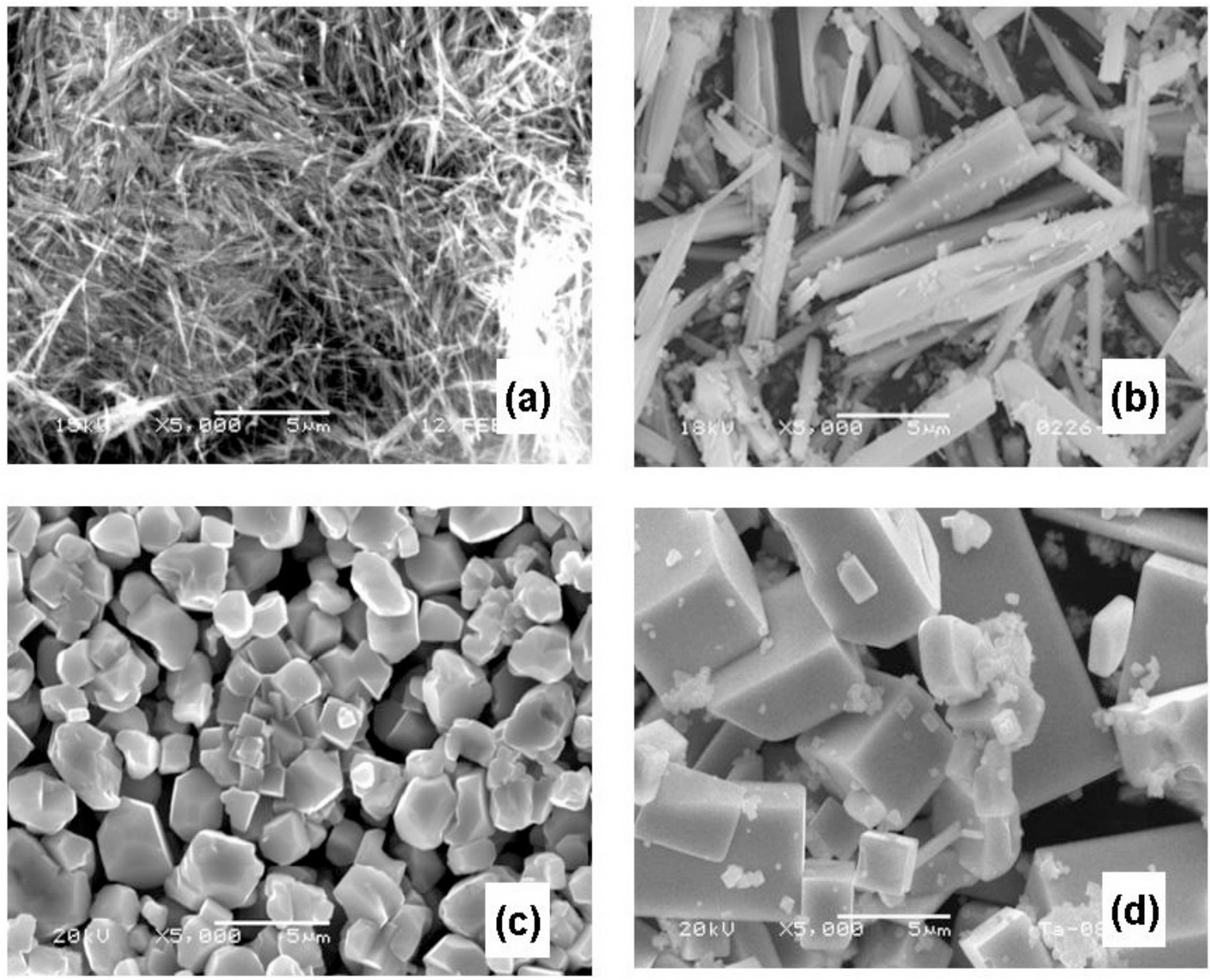

The application of supercritical and subcritical water for the hydrothermal synthesis of multicomponent oxide photocatalysts was successfully demonstrated. $\mathrm{KTiNbO}_{5}$ powders with single phase, rectangular particle shape and large surface area are achieved by the hydrothermal method under subcritical and supercritical conditions. This novel process shows many advantages such as lowering 
the reaction temperature, regular fine particles morphology and high photocatalytic performances in comparison with the conventional solid state methods for synthesizing $\mathrm{KTiNbO}_{5}$ powders. The hydrothermally synthesized $\mathrm{KTiNbO}_{5}$ powders with Ru loading exhibit photocatalytic activity of 7-12 fold higher than that prepared by the solid-state method in the decomposition of aqueous methanol solution. Large surface area of $\mathrm{KTiNbO}_{5}$ powders has a principal effect on the photocatalytic activity of $\mathrm{Ru}$ loaded $\mathrm{KTiNbO}_{5}$ photocatalyst [8-10].

Table 1. Particle formation using supercritical hydrothermal batch reaction system.

\begin{tabular}{|c|c|c|c|c|}
\hline Starting Materials & Conditions & Products & Particle size (nm) & Reference \\
\hline $\mathrm{Ti}\left(\mathrm{OC}_{3} \mathrm{H}_{7}\right)_{4}$ & $100-400^{\circ} \mathrm{C}, 24 \mathrm{~h}$ & $\mathrm{TiO}_{2}$ & $20-115$ & [4] \\
\hline $\mathrm{Ti}\left(\mathrm{OC}_{3} \mathrm{H}_{7}\right)_{4}, \mathrm{KOH}$ & $\begin{array}{c}350-450{ }^{\circ} \mathrm{C}, 14-44 \mathrm{MPa}, \\
2-25 \mathrm{~h}\end{array}$ & $\mathrm{~K}_{2} \mathrm{Ti}_{6} \mathrm{O}_{13}$ & $12.5-20.1$ & {$[5]$} \\
\hline $\mathrm{Nb}_{2} \mathrm{O}_{5}, \mathrm{KOH}$ & $\begin{array}{c}200-400{ }^{\circ} \mathrm{C} \\
2-72 \mathrm{~h}\end{array}$ & $\begin{array}{c}\mathrm{K}_{4} \mathrm{Nb}_{6} \mathrm{O}_{19} \\
\mathrm{KNbO}_{3} \\
\end{array}$ & $7-19$ & {$[6]$} \\
\hline $\mathrm{Nb}_{2} \mathrm{O}_{5}, \mathrm{KOH}$ & $\begin{array}{c}400{ }^{\circ} \mathrm{C}, 24 \mathrm{MPa}, \\
2-24 \mathrm{~h}\end{array}$ & $\mathrm{KNbO}_{3}$ & $15.2-42.1$ & {$[7]$} \\
\hline $\begin{array}{l}\mathrm{Ti}\left(\mathrm{OC}_{3} \mathrm{H}_{7}\right)_{4} \\
\mathrm{Nb}_{2} \mathrm{O}_{5}, \mathrm{KOH}\end{array}$ & $\begin{array}{c}300-400{ }^{\circ} \mathrm{C}, 3-25 \mathrm{MPa} \\
2-24 \mathrm{~h}\end{array}$ & $\mathrm{KTiNbO}_{5}$ & $1000-3000$ & [8-10] \\
\hline $\mathrm{Ta}_{2} \mathrm{O}_{5}, \mathrm{KOH}$ & $\begin{array}{c}400{ }^{\circ} \mathrm{C}, 25 \mathrm{MPa}, \\
2-48 \mathrm{~h}\end{array}$ & $\mathrm{KTaO}_{3}$ & $1000-10000$ & [11] \\
\hline $\begin{array}{l}\mathrm{Zn}\left(\mathrm{NO}_{3}\right)_{2}, \\
\mathrm{Mn}\left(\mathrm{NO}_{3}\right)_{2} \mathrm{SiO}_{2}, \\
\mathrm{KOH}\end{array}$ & $\begin{array}{c}400{ }^{\circ} \mathrm{C}, 29 \mathrm{MPa}, \\
0.5-1.5 \mathrm{~h}\end{array}$ & $\mathrm{Zn}_{2} \mathrm{SiO}_{4}: \mathrm{Mn}$ & $\begin{array}{c}\text { Several } \mu \mathrm{m} \text { in } \\
\text { length } \\
\text { (rod-like) }\end{array}$ & {$[12-16]$} \\
\hline $\begin{array}{l}\mathrm{Fe}\left(\mathrm{NO}_{3}\right)_{3} 9 \mathrm{H}_{2} \mathrm{O} \\
\mathrm{In}\left(\mathrm{NO}_{3}\right)_{3} 5 \mathrm{H}_{2} \mathrm{O}\end{array}$ & $400{ }^{\circ} \mathrm{C}, 30 \mathrm{MPa}, 4 \mathrm{~h}$ & $\begin{array}{l}-\left(\mathrm{Fe}_{1-\mathrm{x}} \mathrm{In}_{\mathrm{x}}\right)_{2} \mathrm{O}_{3} \\
\mathrm{c}-\left(\mathrm{Fe}_{1-\mathrm{x}} \mathrm{In}_{\mathrm{x}}\right)_{2} \mathrm{O}_{3}\end{array}$ & $30-40$ & {$[32]$} \\
\hline $\mathrm{FeSO}_{4} 7 \mathrm{H}_{2} \mathrm{O}, \mathrm{LiOH}$ & $\begin{array}{c}121-388^{\circ} \mathrm{C},<33.5 \mathrm{MPa} \\
10 \mathrm{~min}-1 \mathrm{~h}\end{array}$ & $\mathrm{LiFePO}_{4}$ & $1000-2000$ & {$[33]$} \\
\hline $\begin{array}{l}\mathrm{Ce}\left(\mathrm{NO}_{3}\right)_{3}, \\
\text { lignosulfate }\end{array}$ & $250^{\circ} \mathrm{C}, 10 \mathrm{~min}$ & $\mathrm{CeO}_{2}$ & $5-20$ & {$[34]$} \\
\hline $\begin{array}{l}\mathrm{V}_{2} \mathrm{O}_{5}, \mathrm{Y}\left(\mathrm{NO}_{3}\right)_{3}, \\
\mathrm{KOH}\end{array}$ & $200-380^{\circ} \mathrm{C}, 1-10 \mathrm{~h}$ & $\mathrm{YVO}_{4}$ & $50-200$ & {$[35]$} \\
\hline $\begin{array}{l}\mathrm{Co}\left(\mathrm{NO}_{3}\right)_{2} 6 \mathrm{H}_{2} \mathrm{O} \\
\mathrm{Fe}\left(\mathrm{NO}_{3}\right)_{3} 9 \mathrm{H}_{2} \mathrm{O} \\
(\mathrm{Li}, \mathrm{Na}, \mathrm{K}) \mathrm{OH}\end{array}$ & $390^{\circ} \mathrm{C}, \mathrm{pH} 12<6 \mathrm{~h}$ & $\mathrm{CoFe}_{2} \mathrm{O}_{4}$ & 5 & {$[36]$} \\
\hline $\mathrm{Fe}\left(\mathrm{NO}_{3}\right)_{3} 9 \mathrm{H}_{2} \mathrm{O}$ & $394^{\circ} \mathrm{C}, 5-30 \mathrm{~d}$ & $\mathrm{Fe}_{2} \mathrm{O}_{3}$ & $16-36$ & [37] \\
\hline $\begin{array}{l}\mathrm{Y}\left(\mathrm{NO}_{3}\right)_{3} 6 \mathrm{H}_{2} \mathrm{O} \\
\mathrm{Al}\left(\mathrm{NO}_{3}\right)_{3} 9 \mathrm{H}_{2} \mathrm{O} \\
\mathrm{Eu}\left(\mathrm{NO}_{3}\right)_{3} 6 \mathrm{H}_{2} \mathrm{O} \\
\mathrm{KOH}\end{array}$ & $\begin{array}{c}400{ }^{\circ} \mathrm{C}, 30 \mathrm{MPa}, \\
2 \mathrm{~h}, \mathrm{pH} 7-11\end{array}$ & $\begin{array}{l}\mathrm{Y}_{3} \mathrm{Al}_{5} \mathrm{O}_{12} \\
(\mathrm{YAG}): \mathrm{Eu}\end{array}$ & $\begin{array}{l}\quad<3000 \text { in } \\
\text { length(rod-like) }\end{array}$ & {$[38]$} \\
\hline $\begin{array}{l}\mathrm{Ce}\left(\mathrm{NO}_{3}\right)_{3} 6 \mathrm{H}_{2} \mathrm{O}, \\
\mathrm{NaOH} \\
\end{array}$ & $\begin{array}{c}390^{\circ} \mathrm{C}, \mathrm{pH} 7-9 \\
2 \mathrm{~h} \\
\end{array}$ & $\mathrm{CeO}_{2}$ & $3-8$ & [39] \\
\hline $\mathrm{Er}_{2} \mathrm{O}_{3}, \mathrm{NaOH}$ & $\begin{array}{c}300{ }^{\circ} \mathrm{C}, 25 \mathrm{MPa} \\
2-22 \mathrm{~h}\end{array}$ & $\begin{array}{c}\mathrm{ErOOH} \\
\mathrm{Er}_{2} \mathrm{OCO}_{3}(\mathrm{OH})_{2} \\
\end{array}$ & $\begin{array}{c}6000-12000 \\
\text { (rod-like) }\end{array}$ & {$[40]$} \\
\hline $\begin{array}{l}\mathrm{MgCl}_{2} 6 \mathrm{H}_{2} \mathrm{O} \\
\mathrm{K}_{4} \mathrm{P}_{2} \mathrm{O}_{7}, \mathrm{HCl} \\
\end{array}$ & $\begin{array}{c}400-450{ }^{\circ} \mathrm{C}, 25-32 \mathrm{MPa} \\
5-120 \mathrm{~min}\end{array}$ & $\mathrm{Mg}_{3.5} \mathrm{H}_{2}\left(\mathrm{PO}_{4}\right)_{3}$ & $20-500$ & {$[41]$} \\
\hline $\begin{array}{l}\mathrm{Cu}\left(\mathrm{NO}_{3}\right)_{2} 3 \mathrm{H}_{2} \mathrm{O}, \\
\mathrm{Al}\left(\mathrm{NO}_{3}\right)_{3} 9 \mathrm{H}_{2} \mathrm{O}, \\
\mathrm{HCOOH}, \mathrm{NaOH}^{-} \mathrm{O}\end{array}$ & $\begin{array}{c}400{ }^{\circ} \mathrm{C}, 30 \mathrm{MPa}, \\
10-30 \mathrm{~min}\end{array}$ & $\mathrm{CuAlO}_{2}$ & $2000-5000$ & {$[42]$} \\
\hline
\end{tabular}


In the batch reaction system, one of the advantages for synthesis of metal oxides in supercritical water is reducing alkaline concentration for the crystal phase formation. As mentioned above, potassium niobate can be synthesized under supercritical conditions. High concentration of $\mathrm{KOH}$ $(>6 \mathrm{M})$ is required for the conventional hydrothermal preparation of $\mathrm{KNbO}_{3}\left(<200{ }^{\circ} \mathrm{C}\right)$ [29]. High alkalinity conditions usually causes serious corrosion of reaction vessel and results in more difficult waste treatment, thereby making it an obstacle for industrial manufacture. $\mathrm{KNbO}_{3}$ ceramic particles were successfully prepared by the glycothermal method using $0.5 \mathrm{M} \mathrm{KOH}$ in supercritical isopropanol [30]. Although the required concentration of $\mathrm{KOH}$ in the reaction was reduced, isopropanol is flammable, toxic, and may cause serious pollution. In contrast, supercritical water is an environmentally benign fluid, thus its application in the hydrothermal process has attracted much attention. Supercritical water can be categorized as solvothermal reaction, because properties of water such as viscosity, dielectric constant and solubility for many compounds will change drastically. Synthesis of $\mathrm{KNbO}_{3}$ powders in supercritical water employed significantly low $\mathrm{KOH}$ concentrations $(0.1-0.5 \mathrm{M})$, which was far less than the very high concentrations required for the conventional hydrothermal method for preparing $\mathrm{KNbO}_{3}$ powders. Structure characterization results indicated that the $\mathrm{KNbO}_{3}$ powders prepared in supercritical water had rhombohedral and orthorhombic structures (Figure 2c) depending on the $\mathrm{KOH}$ concentrations. Potassium niobate is a kind of famous ferroelectric materials, which has promising applications in non-linear optical device. The orthorhombic form of $\mathrm{KNbO}_{3}$ powder prepared in supercritical water exhibited a strong second harmonic generation (SHG) activity of non-linear optical property which is similar intensity of the solid-state synthesized $\mathrm{KNbO}_{3}$ [7].

Similar to the $\mathrm{KNbO}_{3}$, perovskite $\mathrm{KTaO}_{3}$ crystals have been successfully prepared by hydrothermal reaction in 0.34-1.0 M KOH solution under supercritical conditions. Morphology of $\mathrm{KTaO}_{3}$ crystals tends to a rectangular shape ranging in size from 10 to $100 \mathrm{~nm}$ (Figure 2d). Using supercritical water, the $\mathrm{KOH}$ concentration required to form the perovskite phase is far lower $(<0.5 \mathrm{M})$ than that previously reported for conventional hydrothermal conditions $(>7.0 \mathrm{M})$ [31]. Solvothermal preparation of $\mathrm{KTaO}_{3}$ particles was reported using 1.0 M KOH ion water-ethanol mixed solvents. Reactions under solvothermal conditions could happen easier than those under conventional hydrothermal conditions because of the low dielectric constant. In addition, epitaxial crystal growth of $\mathrm{KTaO}_{3}$ can be successfully achieved on the $\mathrm{SrTiO}_{3}$ substrate even under supercritical conditions. $\mathrm{KTaO}_{3}$ films were achieved on the (100) $\mathrm{SrTiO}_{3}$ substrate in $0.5 \mathrm{M} \mathrm{KOH}$ aqueous solution under supercritical conditions. The $\mathrm{KTaO}_{3}$ grew epitaxially on a (100) oriented single crystal $\mathrm{SrTiO}_{3}$ substrate. Electron backscatter patterns for the $\mathrm{KTaO}_{3}$ crystals supported that the $\mathrm{KTaO}_{3}$ crystals grow in the (001) [100] orientation which is the same as $\mathrm{SrTiO}_{3}$ substrate [11].

Another advantage for synthesis of metal oxides in supercritical water is reducing process energy for the production of highly crystalline particles. Mn-doped zinc silicate, $\alpha-\mathrm{Zn}_{2} \mathrm{SiO}_{4}: \mathrm{Mn}^{2+}$ is a practical inorganic phosphor and consumed in large volume as a green phosphor for plasma-display panels. Commercial zinc silicate phosphor is produced by a solid-state reaction at temperatures higher than $1000{ }^{\circ} \mathrm{C}$ and for processing times of several hours to provide irregularly shaped particles with several to several tens of microns. To improve morphology of zinc silicate and to establish lowtemperature processing routes, supercritical method can produce $\alpha-\mathrm{Zn}_{2} \mathrm{SiO}_{4}: \mathrm{Mn}^{2+}$ without post calcinations, indicating that crystallization of $\alpha-\mathrm{Zn}_{2} \mathrm{SiO}_{4}: \mathrm{Mn}^{2+}$ in solvent occurs at temperatures lower 
than those required in solid-diffusion. $\alpha$-phase $\mathrm{Zn}_{2} \mathrm{SiO}_{4}: \mathrm{Mn}^{2+}$ can be synthesized under supercritical conditions at $400{ }^{\circ} \mathrm{C}, 29 \mathrm{MPa}$ and reaction time of $90 \mathrm{~min}$, which had an equivalent luminescence as that produced by the same raw materials with a firing process at $1200{ }^{\circ} \mathrm{C}$ for $240 \mathrm{~min}$ [12]. Products synthesized by a supercritical water method had rod-like shaped $\alpha$-phase $\mathrm{Zn}_{2} \mathrm{SiO}_{4}: \mathrm{Mn}^{2+}$ particles with lengths of 2000-9000 nm and widths of 500-1000 nm (Figure 2b). Supercritical water provides a dense crystal of $\mathrm{Zn}_{2} \mathrm{SiO}_{4}$ within a very short reaction time due to the reaction medium being at higher pressure than is usual for hydrothermal and solvothermal conditions. Thus, supercritical hydrothermal process has the potential to be used in a practical process to produce highly crystalline $\mathrm{Zn}_{2} \mathrm{SiO}_{4}$ particles with low environmental burden [13-16].

Although the batch reaction process is a useful technique for the synthesis of highly crystalline powders and large single crystals, the reaction takes a long time including heating and cooling steps depending on the reactor volume. In contrast, flow reaction system is a promising process for production of nano-sized metal oxide particles, since rapid heating and cooling can be controlled and short reaction time can be achieved under continuous operating conditions.

\subsection{Flow Reaction System}

The flow reaction system exhibits two features in addition to the supercritical hydrothermal batch reaction system. Firstly, a sudden change in the dielectric constant of the reaction medium results in a high-density of homogeneous nucleation. Secondly, a very short reaction time of less than 10 seconds can suppress crystal growth and aggregation. Thus, the rapid and continuous synthesis of single nanosized metal oxides with a relatively narrow size distribution is possible. The conversion, particle size, and crystallinity were analyzed for some metal oxide particles on the basis of metal oxide solubility and supersaturation. As solubility decreased, the conversion increased in the order: $\mathrm{ZrO}_{2}>\mathrm{Fe}_{2} \mathrm{O}_{3}>$ $\mathrm{AlOOH} / \mathrm{Al}_{2} \mathrm{O}_{3}>\mathrm{NiO}>\mathrm{CuO}$. Namely, the solubility strongly depended on the precipitation rate. The relationship between the average particle size and supersaturation is widely known in traditional nucleation theory; average particle size tends to decrease with increasing supersaturation. It was found that supersaturation higher than a factor of about $10^{4}$ was needed to obtain particles under $10 \mathrm{~nm}$ in diameter. Addition of $\mathrm{KOH}$ tends to cause the particle size to decrease, which can be attributed to the decrease in solubility. Condition of high supersaturation means that the driving force for precipitation is large and during this non-steady precipitation process, water molecules are easily occluded in precipitates. As a result, low crystalline solids can be produced. In contrast, small supersaturation generally leads to slower precipitation and during this period relatively stable intermediate species are probably formed. For these differences, it was assumed that crystallinity increased with decreasing supersaturation [28].

The starting materials, synthetic conditions, product phases and particle sizes of the materials obtained by supercritical hydrothermal flow reaction system are described in Table 2 and transmission electron micrographs (TEM) of representative particles are shown in Figure 3. Particle properties are dominated by significant parameters such as temperature, pressure, reaction time, and reactant concentrations. 
Table 2. (1). Particle formation using the supercritical hydrothermal flow reaction system.

(2). Particle formation using the supercritical hydrothermal flow reaction system.

(1)

\begin{tabular}{|c|c|c|c|c|}
\hline Starting Materials & Conditions & Products & Particle size (nm) & Reference \\
\hline $\mathrm{TiO}_{2}$ sol, $\mathrm{KOH}$ & $\begin{array}{c}350-420{ }^{\circ} \mathrm{C} \\
30 \mathrm{MPa}, 2-3 \mathrm{~s}\end{array}$ & $\begin{array}{c}\mathrm{K}_{2} \mathrm{Ti}_{6} \mathrm{O}_{13} \\
\mathrm{TiO}_{2}\end{array}$ & $\begin{array}{c}10 \text { (width), } \\
500-1000 \text { (length) }\end{array}$ & {$[17,18]$} \\
\hline $\begin{array}{l}\mathrm{ZrO}\left(\mathrm{NO}_{3}\right)_{2} \\
\mathrm{ZrO}(\mathrm{Ac})_{2}\end{array}$ & $\begin{array}{c}400{ }^{\circ} \mathrm{C}, 30 \mathrm{MPa}, \\
1.8 \mathrm{~s}\end{array}$ & $\mathrm{ZrO}_{2}$ & $6.8-7$ & {$[19,27,28]$} \\
\hline $\mathrm{Al}\left(\mathrm{NO}_{3}\right)_{3}$ & $\begin{array}{c}350-400{ }^{\circ} \mathrm{C}, \\
25-40 \mathrm{MPa}, 2-64 \mathrm{~s}\end{array}$ & $\gamma-\mathrm{AlOOH}$ & $63-473$ & {$[20]$} \\
\hline $\mathrm{Al}\left(\mathrm{NO}_{3}\right)_{3}$ & $\begin{array}{c}400-500{ }^{\circ} \mathrm{C}, \\
25-35 \mathrm{MPa}, 0.063-3 \mathrm{~s}\end{array}$ & $\begin{array}{c}\gamma-\mathrm{AlOOH} \\
\gamma-\mathrm{Al}_{2} \mathrm{O}_{3} \\
\end{array}$ & $3.9-6.4$ & [21] \\
\hline $\mathrm{Ba}(\mathrm{OH})_{2}, \mathrm{TiO}_{2}$ sol & $\begin{array}{c}300-420{ }^{\circ} \mathrm{C}, 30 \mathrm{MPa}, \\
0.1-40 \mathrm{~s}\end{array}$ & $\mathrm{BaTiO}_{3}$ & $13-48.4$ & {$[22]$} \\
\hline $\mathrm{Ba}(\mathrm{OH})_{2}, \mathrm{TiO}_{2}$ sol & $\begin{array}{c}300-420^{\circ} \mathrm{C} \\
20-40 \mathrm{MPa}, 0.7-5.1 \mathrm{~s}\end{array}$ & $\begin{array}{c}\text { Tetragonal/ } \\
\text { Cubic } \mathrm{BaTiO}_{3}\end{array}$ & $10-100$ & [23] \\
\hline $\mathrm{Ba}(\mathrm{OH})_{2}, \mathrm{TiO}_{2}$ sol & $\begin{array}{c}400{ }^{\circ} \mathrm{C}, 30 \mathrm{MPa}, \\
7 \mathrm{~ms}-2 \mathrm{~s}\end{array}$ & $\begin{array}{c}\text { Tetragonal/ } \\
\text { Cubic } \mathrm{BaTiO}_{3}\end{array}$ & $9-32$ & {$[24]$} \\
\hline $\begin{array}{l}\mathrm{Ca}\left(\mathrm{NO}_{3}\right)_{2}, \\
\mathrm{Sr}\left(\mathrm{NO}_{3}\right)_{2}, \\
\mathrm{Fe}\left(\mathrm{NO}_{3}\right)_{3}, \\
\mathrm{TiO}_{2} \mathrm{sol}\end{array}$ & $\begin{array}{l}300-400{ }^{\circ} \mathrm{C} \\
30 \mathrm{MPa}, 10 \mathrm{~s}\end{array}$ & $\mathrm{Ca}_{0.8} \mathrm{Sr}_{0.2} \mathrm{Ti}_{1 \mathrm{x}} \mathrm{Fe}_{\mathrm{x}} \mathrm{O}_{3-}$ & $20-27$ & {$[25]$} \\
\hline $\begin{array}{l}\mathrm{ZrO}\left(\mathrm{NO}_{3}\right)_{2} \\
\mathrm{Y}\left(\mathrm{NO}_{3}\right)_{3} \\
\end{array}$ & $\begin{array}{c}300-400{ }^{\circ} \mathrm{C}, 30 \mathrm{MPa}, \\
0.17-0.35 \mathrm{~s} \\
\end{array}$ & YSZ & $4-6$ & {$[26]$} \\
\hline $\begin{array}{l}\mathrm{Zn}\left(\mathrm{CH}_{3} \mathrm{CO}_{2}\right)_{2} \\
\mathrm{H}_{2} \mathrm{O}_{2}\end{array}$ & $\begin{array}{c}400^{\circ} \mathrm{C}, 245 \mathrm{~atm}, \\
8.9-16.3 \mathrm{~s} \\
\end{array}$ & $\mathrm{ZnO}$ & $39-320$ & {$[43]$} \\
\hline $\begin{array}{l}\mathrm{Fe}\left(\mathrm{NO}_{3}\right)_{3} \\
\mathrm{Co}\left(\mathrm{NO}_{3}\right)_{2} \\
\mathrm{NaOH} \\
\end{array}$ & $\begin{array}{c}75-675 \mathrm{~K}, 25 \mathrm{MPa} \\
11-23 \mathrm{~s}\end{array}$ & $\mathrm{CoFe}_{2} \mathrm{O}_{4}$ & $13-23$ & {$[45]$} \\
\hline $\begin{array}{l}\mathrm{Al}\left(\mathrm{NO}_{3}\right)_{3} \\
\mathrm{Y}\left(\mathrm{NO}_{3}\right)_{3} \\
\mathrm{~Tb}\left(\mathrm{NO}_{3}\right)_{3}, \\
\mathrm{KOH}\end{array}$ & $\begin{array}{c}400{ }^{\circ} \mathrm{C}, 30 \mathrm{MPa}, \\
2.5 \mathrm{~s}\end{array}$ & $\left(\mathrm{Y}_{2.7} \mathrm{~Tb}_{0.3}\right) \mathrm{Al}_{5} \mathrm{O}_{12}$ & $14-152$ & {$[46]$} \\
\hline $\mathrm{Zn}\left(\mathrm{NO}_{3}\right)_{2} 6 \mathrm{H}_{2} \mathrm{O}$ & $390^{\circ} \mathrm{C}, 30 \mathrm{MPa}, 22 \mathrm{~s}$ & $\mathrm{ZnO}$ & <10000 (whisker) & {$[47]$} \\
\hline $\begin{array}{l}\mathrm{Zn}\left(\mathrm{NO}_{3}\right)_{2} 6 \mathrm{H}_{2} \mathrm{O} \\
\mathrm{LiOH}\end{array}$ & $\begin{array}{c}390^{\circ} \mathrm{C}, 30 \mathrm{MPa}, \\
0.7 \mathrm{~s}\end{array}$ & $\mathrm{ZnO}$ & $16-57$ & {$[48]$} \\
\hline $\begin{array}{l}\mathrm{Zn}\left(\mathrm{NO}_{3}\right)_{2} 6 \mathrm{H}_{2} \mathrm{O}, \\
\mathrm{LiOH}\end{array}$ & $\begin{array}{c}400{ }^{\circ} \mathrm{C}, 30 \mathrm{MPa}, \\
0.03 \mathrm{~s}\end{array}$ & $\mathrm{ZnO}$ (nanorod) & $\begin{array}{l}38 \text { (width), } \\
230 \text { (length) }\end{array}$ & {$[49]$} \\
\hline $\begin{array}{l}\mathrm{Ba}(\mathrm{OiPr})_{2}, \\
\mathrm{Ti}(\mathrm{OiPr})_{4}, \mathrm{EtOH}\end{array}$ & $\begin{array}{c}330-380^{\circ} \mathrm{C}, 16 \mathrm{MPa} \\
119-166 \mathrm{~s}\end{array}$ & Cubic $\mathrm{BaTiO}_{3}$ & $15-36$ & {$[51,52]$} \\
\hline $\begin{array}{l}\mathrm{Ba}, \mathrm{Sr})(\mathrm{OiPr})_{2}, \\
\mathrm{Ti}(\mathrm{OiPr}), \mathrm{EtOH}\end{array}$ & $\begin{array}{c}380^{\circ} \mathrm{C}, 26 \mathrm{MPa}, \\
119-166 \mathrm{~s} \\
\end{array}$ & Cubic $\mathrm{BaTiO}_{3}$ & $<50$ & {$[53,54]$} \\
\hline $\begin{array}{l}\mathrm{Mn}\left(\mathrm{NO}_{3}\right)_{2} 6 \mathrm{H}_{2} \mathrm{O} \\
\mathrm{LiOH}, \mathrm{LiNO}_{3}\end{array}$ & $\begin{array}{c}400-420^{\circ} \mathrm{C}, 30 \mathrm{MPa} \\
10-40 \mathrm{~s}\end{array}$ & $\mathrm{LiMn}_{2} \mathrm{O}_{4}$ & $<100$ & {$[55]$} \\
\hline
\end{tabular}


Table 2. Cont.

(2)

\begin{tabular}{|c|c|c|c|c|}
\hline Starting Materials & Conditions & Products & Particle Size (nm) & Reference \\
\hline $\mathrm{SnCl}_{2} \mathrm{InCl}_{3}$ & $\begin{array}{c}350-380^{\circ} \mathrm{C} \\
30 \mathrm{MPa} \\
\end{array}$ & $\begin{array}{c}\text { Cubic/Tetragonal } \mathrm{In}_{2} \mathrm{O}_{3}, \\
\mathrm{SnO}_{2}, \mathrm{ITO}\end{array}$ & $<10$ & {$[57]$} \\
\hline $\begin{array}{l}\mathrm{Al}(\mathrm{AcAc}) / \mathrm{Al}\left(\mathrm{NO}_{3}\right)_{3} \\
\mathrm{Y} \text { acetate } / \mathrm{Y}\left(\mathrm{NO}_{3}\right)_{3}\end{array}$ & $\begin{array}{c}260-385^{\circ} \mathrm{C} \\
24 \mathrm{MPa} \\
\end{array}$ & Cubic $\mathrm{Y}_{2} \mathrm{Al}_{5} \mathrm{O}_{12}$ & $<150$ & {$[58]$} \\
\hline $\begin{array}{l}\mathrm{Al}\left(\mathrm{NO}_{3}\right)_{3}, \mathrm{Y}\left(\mathrm{NO}_{3}\right)_{3}, \\
\mathrm{Eu}\left(\mathrm{NO}_{3}\right)_{3}, \mathrm{KOH}\end{array}$ & $\begin{array}{l}400^{\circ} \mathrm{C} \\
28 \mathrm{MPa}\end{array}$ & Cubic $(\mathrm{Y}, \mathrm{Eu})_{2} \mathrm{Al}_{5} \mathrm{O}_{12}$ & $<100$ & {$[59]$} \\
\hline $\begin{array}{l}\mathrm{La}\left(\mathrm{NO}_{3}\right)_{3}, \\
\mathrm{Ni}\left(\mathrm{NO}_{3}\right)_{2}, \mathrm{KOH}\end{array}$ & $\begin{array}{l}400^{\circ} \mathrm{C} \\
24 \mathrm{MPa}\end{array}$ & $\begin{array}{l}\text { Rhombohedral } \\
\mathrm{La}_{\mathrm{n}+1} \mathrm{Ni}_{\mathrm{n}} \mathrm{O}_{3 \mathrm{n}+1}\end{array}$ & $<430$ & {$[60,76]$} \\
\hline $\begin{array}{l}\mathrm{Zr}\left(\mathrm{CH}_{3} \mathrm{COO}\right)_{4} / \\
\mathrm{Zr}\left(\mathrm{CH}_{3} \mathrm{CH}_{2} \mathrm{O}\right)\end{array}$ & $\begin{array}{c}300-450^{\circ} \mathrm{C}, \\
10-45 \mathrm{MPa}\end{array}$ & $\begin{array}{c}\text { Tetragonal/ } \\
\text { monoclinic } \mathrm{ZrO}_{2}\end{array}$ & $<10$ & {$[67]$} \\
\hline $\mathrm{SnCl}_{2}$ & $\begin{array}{c}385-415^{\circ} \mathrm{C} \\
30 \mathrm{MPa}\end{array}$ & Tetragonal/ $\mathrm{SnO}_{2}$ & $<10$ & {$[68]$} \\
\hline $\mathrm{Al}\left(\mathrm{NO}_{3}\right)_{3}, \mathrm{KOH}$ & $\begin{array}{c}400{ }^{\circ} \mathrm{C} \\
30-40 \mathrm{MPa}\end{array}$ & $\begin{array}{c}\gamma-\mathrm{AlOOH} \\
\gamma-\mathrm{Al}_{2} \mathrm{O}_{3} \\
\end{array}$ & $<20$ & [69] \\
\hline $\mathrm{Fe}\left(\mathrm{NO}_{3}\right)_{3}, \mathrm{PVA}$ & $\begin{array}{c}487-648 \mathrm{~K}, \\
21.7-23 \mathrm{MPa} \\
\end{array}$ & $\alpha-\mathrm{Fe}_{2} \mathrm{O}_{3}$ & $<23$ & {$[70]$} \\
\hline $\mathrm{FeSO}_{4}, \mathrm{H}_{3} \mathrm{PO}_{4}, \mathrm{LiOH}$ & $573-658 \mathrm{~K}$ & $\begin{array}{c}\text { Orthorhombic } \\
\mathrm{LiFePO}_{4} \\
\end{array}$ & $<130$ & {$[71]$} \\
\hline $\mathrm{TiO}_{2}$ sol, $\mathrm{Ba}(\mathrm{OH})_{2}$ & $\begin{array}{l}400^{\circ} \mathrm{C} \\
30 \mathrm{MPa}\end{array}$ & $\begin{array}{l}\text { Tetragonal } \\
\mathrm{BaTiO}_{3}\end{array}$ & $<20$ & {$[72]$} \\
\hline $\begin{array}{l}\mathrm{ZrO}\left(\mathrm{NO}_{3}\right)_{2}, \mathrm{Ba}(\mathrm{OH})_{2} / \\
\mathrm{Ba}\left(\mathrm{NO}_{3}\right)_{2} / \mathrm{Ba}\left(\mathrm{CH}_{3} \mathrm{CO}_{2}\right)_{2}, \\
\mathrm{NaOH}\end{array}$ & $\begin{array}{c}450-485^{\circ} \mathrm{C} \\
30 \mathrm{MPa}\end{array}$ & Cubic $\mathrm{BaZrO}_{3}$ & $<100$ & {$[73]$} \\
\hline $\begin{array}{l}\mathrm{Fe}\left(\mathrm{NO}_{3}\right)_{3}, \mathrm{Ni}\left(\mathrm{NO}_{3}\right)_{2}, \\
\mathrm{Cu}\left(\mathrm{NO}_{3}\right)_{2}, \mathrm{Zn}\left(\mathrm{NO}_{3}\right)_{2}, \\
\mathrm{KOH}\end{array}$ & $\begin{array}{l}400^{\circ} \mathrm{C} \\
30 \mathrm{MPa}\end{array}$ & $\begin{array}{c}\text { Rhombohedral/Cubic, } \\
\text { Tetragonal } \\
(\mathrm{Ni}, \mathrm{Cu}, \mathrm{Zn}) \mathrm{Fe}_{2} \mathrm{O}_{4}\end{array}$ & $<22$ & {$[74]$} \\
\hline $\begin{array}{l}\mathrm{Ca}\left(\mathrm{NO}_{3}\right)_{2}, \mathrm{Mg}\left(\mathrm{NO}_{3}\right)_{2}, \\
\left(\mathrm{NH}_{4}\right)_{2} \mathrm{HPO}_{4}\end{array}$ & $\begin{array}{l}400^{\circ} \mathrm{C} \\
30 \mathrm{MPa}\end{array}$ & $\begin{array}{c}\mathrm{Ca}_{10-\mathrm{x}} \mathrm{Mg}_{\mathrm{x}}\left(\mathrm{PO}_{4}\right)_{6}(\mathrm{OH})_{2} / \\
\mathrm{Ca}_{3-\mathrm{y}} \mathrm{Mg}_{\mathrm{y}}\left(\mathrm{HPO}_{4}\right)_{2} \\
\left(\mathrm{PO}_{4}\right)_{2-2 \mathrm{x} / 3}\end{array}$ & $<80$ & {$[75]$} \\
\hline $\begin{array}{l}\mathrm{ZrO}\left(\mathrm{NO}_{3}\right)_{2}, \mathrm{Ce}\left(\mathrm{NO}_{3}\right)_{3}, \\
\mathrm{NH}_{4} \mathrm{OH}\end{array}$ & $\begin{array}{l}\text { Supercritical } \\
\text { Conditions }\end{array}$ & $\begin{array}{l}\text { Cubic/Tetragonal } \\
\mathrm{Ce}_{\mathrm{x}} \mathrm{Zr}_{1-\mathrm{x}} \mathrm{O}_{2}\end{array}$ & $7-16$ & {$[77]$} \\
\hline $\begin{array}{l}\mathrm{Co}\left(\mathrm{NO}_{3}\right)_{2}, \mathrm{KOH}, \\
\mathrm{Ni}\left(\mathrm{NO}_{3}\right)_{2} / \\
\mathrm{Ni}\left(\mathrm{CH}_{3} \mathrm{CO}_{2}\right)_{2} \mathrm{H}_{2} \mathrm{O}_{2} \\
\end{array}$ & $\begin{array}{l}90-310^{\circ} \mathrm{C} \\
24.1 \mathrm{MPa}\end{array}$ & $\begin{array}{c}\text { Hexagonal, Cubic } \\
\mathrm{Ni}(\mathrm{OH})_{2} \mathrm{Co}_{\mathrm{x}} \mathrm{Ni}_{1-\mathrm{x}}(\mathrm{OH})_{2} \\
\mathrm{NiCo}_{2} \mathrm{O}_{4}\end{array}$ & $<100$ & {$[78]$} \\
\hline $\begin{array}{l}\mathrm{Zn}\left(\mathrm{NO}_{3}\right)_{2}, \mathrm{KOH}, \\
\text { hexylamine }\end{array}$ & $\begin{array}{l}400^{\circ} \mathrm{C} \\
30 \mathrm{MPa}\end{array}$ & Hexagonal $\mathrm{ZnO}$ & $<150 \times 600($ rod $)$ & {$[79]$} \\
\hline $\begin{array}{l}\mathrm{Ce}\left(\mathrm{NO}_{3}\right)_{3}, \\
\text { Hexanoic acid }\end{array}$ & $\begin{array}{l}250^{\circ} \mathrm{C} \\
25 \mathrm{MPa}\end{array}$ & Cubic $\mathrm{CeO}_{2}$ & $<60$ & {$[80]$} \\
\hline $\begin{array}{l}\mathrm{Ce}\left(\mathrm{NO}_{3}\right)_{3}, \\
\text { Decanoic acid } \\
(\text { MetOH })\end{array}$ & $\begin{array}{l}400^{\circ} \mathrm{C} \\
30 \mathrm{MPa}\end{array}$ & Cubic $\mathrm{CeO}_{2}$ & $<50$ & {$[81]$} \\
\hline
\end{tabular}


As mentioned in the batch reaction system, potassium hexatitanate fibers can be synthesized under subcritical and supercritical conditions for several hours of heating time. The reaction time affects phase formation and the particle size of $\mathrm{K}_{2} \mathrm{Ti}_{6} \mathrm{O}_{13}$. The potassium titanate fibers were synthesized using the flow type hydrothermal reaction system. Under subcritical conditions (at $350{ }^{\circ} \mathrm{C}$ ), a mixture of $\mathrm{TiO}_{2}$ particles and KTO fibers was obtained. Only fibrous KTO single phase was achieved under supercritical conditions even in a short reaction time (2-3 s). The conventional hydrothermal method using a batch reactor produces micron-order KTO fibers (Figure 2a) in supercritical water for a reaction lasting several hours. In contrast, the flow reaction system could produce nano-scaled KTO fibers (Figure 3b) in several seconds, even at nearly the same temperature and pressure, since an extremely short heating time of the flow reaction system can complete crystallization of KTO nanofibers. The hydrogen evolution rate on KTO synthesized using the flow reaction system from photodecomposition of methanol was 10-times greater than those of KTO fibers with micron size, which were synthesized by a supercritical hydrothermal reaction at $400{ }^{\circ} \mathrm{C}$ and $28 \mathrm{MPa}$ for 24 hours. The KTO nano-fibers synthesized by hydrothermal flow reaction exhibited high photocatalytic activity since KTO nano-fibers were well dispersed into water-methanol solution and not precipitated as well as the large BET surface area $\left(220 \mathrm{~m}^{2} / \mathrm{g}\right)$ compared with those of solid-state synthesized KTO $\left(<5 \mathrm{~m}^{2} / \mathrm{g}\right)$ and supercritical batch synthesized KTO $\left(20 \mathrm{~m}^{2} / \mathrm{g}\right)[17,18]$.

Figure 3. Transmission electron microscopy (TEM) images of metal oxide nano-particles synthesized by the supercritical hydrothermal flow system. (a) $\mathrm{Y}_{\mathrm{x}} \mathrm{Zr}_{1-\mathrm{x}} \mathrm{O}_{2}$, (b) $\mathrm{K}_{2} \mathrm{Ti}_{6} \mathrm{O}_{13}$, (c) $\mathrm{BaTiO}_{3}$, (d) $\mathrm{Ca}_{0.7} \mathrm{Sr}_{0.3} \mathrm{Ti}_{0.9} \mathrm{Fe}_{0.1} \mathrm{O}_{3}$.
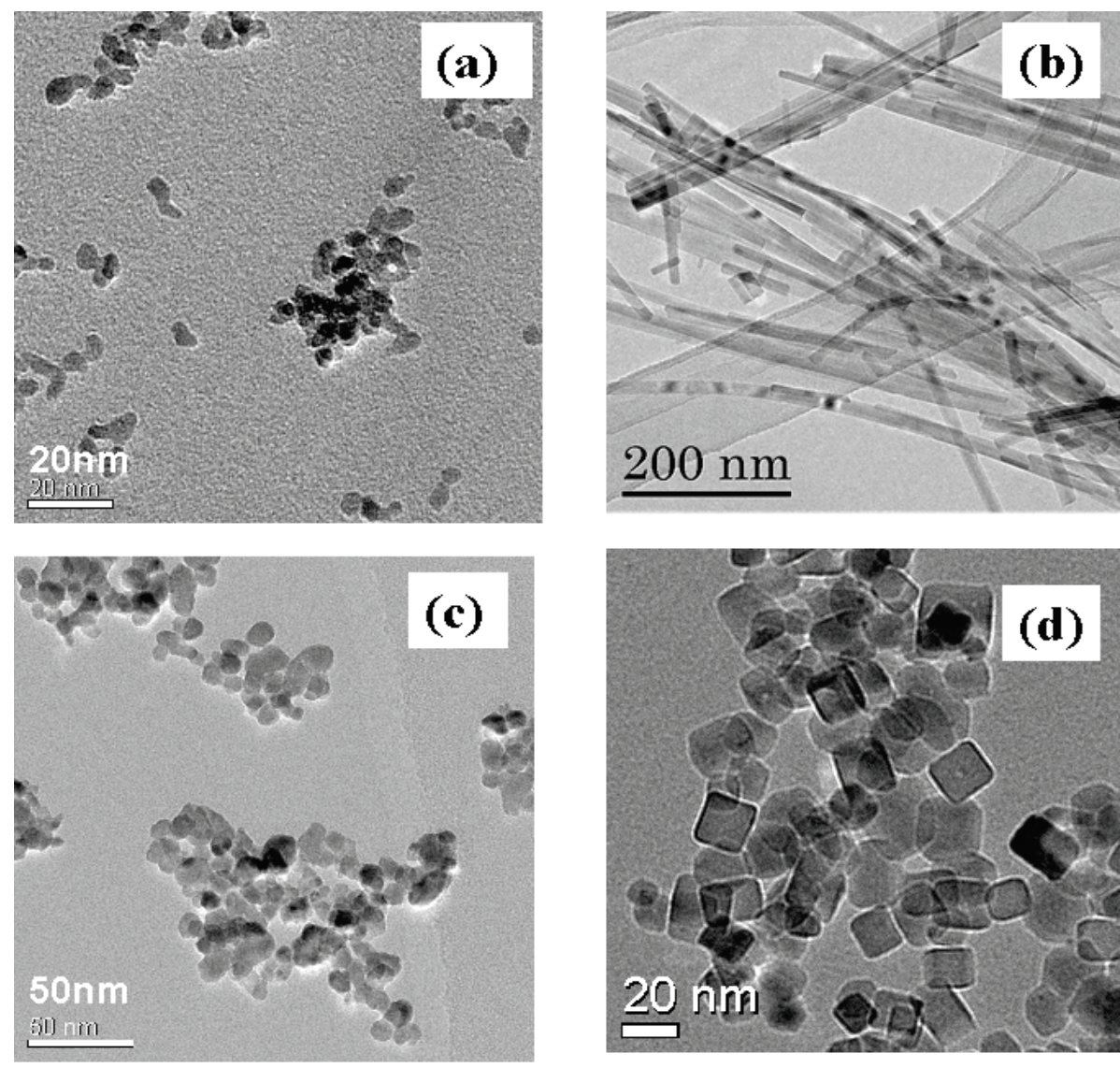
Zirconia particles were prepared by hydrothermal reaction in supercritical water of $400{ }^{\circ} \mathrm{C}$ and $30 \mathrm{MPa}$ from zirconyl salts solution. The hydrothermal reaction went to completion in $1.8 \mathrm{~s}$ and crystalline zirconia particles were produced. Crystal structure of the products depended on a kind of starting metal salts. Stable monoclinic zirconia was formed from zirconyl nitrate solution and a mixture of monoclinic and tetragonal zirconia was obtained from zirconyl acetate solution. Zirconia particles obtained by the hydrothermal synthesis in supercritical water were individual nano-crystals with narrow particle size distribution of 5-8 $\mathrm{nm}$ [19].

Boehmite $(\gamma-\mathrm{AlO}(\mathrm{OH}))$ was synthesized by hydrothermal flow reaction system in a temperature of $350-400{ }^{\circ} \mathrm{C}$. The morphology of the $\gamma-\mathrm{AlO}(\mathrm{OH})$ particles was rhombic or hexagonal plates with $70-470 \mathrm{~nm}$ of the average particle size. The particle size increased with an increase in the reaction temperature and the concentration of starting $\mathrm{Al}\left(\mathrm{NO}_{3}\right)_{3}$ solution, while that decreased with an increase in the $\mathrm{pH}$ of the starting solution. As an effect of reaction pressure on the particle size, the particle size is enlarged from $170 \mathrm{~nm}$ to $300 \mathrm{~nm}$ with increasing reaction pressure from $25 \mathrm{MPa}$ to $40 \mathrm{MPa}$ at $400{ }^{\circ} \mathrm{C}$, whereas there is no dependency of reaction pressure on the particle size at $350{ }^{\circ} \mathrm{C}$. Based on the solubility calculation, the dependencies of the synthetic parameters such as temperature and $\mathrm{pH}$ on the particle size can be explained by an assumption of the separate heating, reacting and cooling regions. Thus, the particle sizes are determined not only by the solubility of the given reaction condition but also by that of the heating or the cooling period [20].

Yttria stabilized zirconia nanoparticles were prepared by hydrothermal flow reaction system under $30 \mathrm{MPa}$ in the temperature range $300-400{ }^{\circ} \mathrm{C}$ and $\mathrm{pH}$ range $1-11$. The yttrium conversion increased with an increase in solution $\mathrm{pH}$ and hydrothermal temperature. Stoichiometric $\mathrm{Y} / \mathrm{Zr}$ solid solution can be achieved at $\mathrm{pH}>8$. Hydrolysis of $\mathrm{Zr}(\mathrm{IV})$ occurs in strongly acidic solutions and zirconium hydrous oxides are precipitated near $\mathrm{pH}$ 2. In contrast, hydrolysis of yttrium does not become appreciable until fairly high $\mathrm{pH}$ values are reached $(>6)$. Accordingly, $\mathrm{pH}$ is a key factor to dope yttrium into zirconia particles stoichiometrically. Primary particle sizes were in the range 4-6 nm irrespective of solution $\mathrm{pH}$ and hydrothermal temperature (Figure 3a) with a relatively narrow particle size distribution owing to large supersaturation of zirconia as already mentioned above [26].

Single phase of perovskite oxide $\mathrm{Ca}_{0.8} \mathrm{Sr}_{0.2} \mathrm{Ti}_{1-\mathrm{x}} \mathrm{FeO}_{3}$ (CTO) nanoparticles were successfully synthesized by a flow supercritical reaction system, in which $\mathrm{Ca}$ and $\mathrm{Ti}$ sites were partially doped by $\mathrm{Sr}$ and $\mathrm{Fe}$ atoms simultaneously. The $\mathrm{pH}$ is the key factor to dope Fe into perovskite oxide completely. By adjusting the $\mathrm{pH}$, the $\mathrm{Sr}$ and $\mathrm{Fe}$ atoms were doped into $\mathrm{Ca}$ and $\mathrm{Ti}$ sites of perovskite oxide, producing single phase CTO. Highly crystalline and uniform CTO nanoparticles with an average particle size of about $20 \mathrm{~nm}$ (Figure 3d) and BET surface area of more than $70 \mathrm{~m}^{2} / \mathrm{g}$ can be produced [25]. The CTO is one of the candidate materials of ceramic membrane reactor for natural gas conversion which can be used for catalyst support and oxygen ionic and electronic conducting membrane. Compared with the conventional solid state reaction and polymeric citrate precursor methods, the fabrication process from powders to membrane can be controlled, since sintering temperature of the membrane decreases and the membrane density increases owing to the smaller particle size and narrow particle size distribution of supercritical hydrothermal synthesized CTO nanoparticles.

In the flow reaction system, as one of the features for synthesis of metal oxides in supercritical water, density of water can be varied with the temperature and pressure under supercritical conditions. 
The crystallite phase can be controlled with the density of water [21,22]. Density of water affects the dehydroxylation from metal hydroxides to metal oxides. Dehydroxylation proceeds at lower density of water under supercritical conditions. One step synthesis of $\gamma-\mathrm{Al}_{2} \mathrm{O}_{3}$ was achieved at $410{ }^{\circ} \mathrm{C}$ or higher temperature in supercritical water. Figure 4 summarizes the relationship between water density and the crystal phase of aluminum oxide. Crystal phase of the products depended not only on reaction temperature but also on reaction pressure. Water density under supercritical conditions is smaller than that of subcritical conditions. The formation of $\gamma-\mathrm{Al}_{2} \mathrm{O}_{3}$ from $\gamma$-AlOOH became dominant because the dehydration reaction was promoted due to decrease of water density from 0.36 to $0.25 \mathrm{~g} / \mathrm{cm}^{3}$ by a temperature of only $10{ }^{\circ} \mathrm{C}$. Primary particle size was unchanged in the range from 4 to $6 \mathrm{~nm}$ regardless of the reaction temperature and reaction time. In addition, dispersed particle size in solution was decreased with the decreasing of $\mathrm{pH}$ and matched with the primary particle size at $\mathrm{pH}$ of 1 or lower owing to protonation for avoiding aggregation of particles [21].

Figure 4. The relationship between $\mathrm{Al}$ oxide polymorph and hydrothermal reaction conditions. Open circle: $\gamma-\mathrm{AlOOH}$, Closed circle: $\gamma-\mathrm{Al}_{2} \mathrm{O}_{3}$.

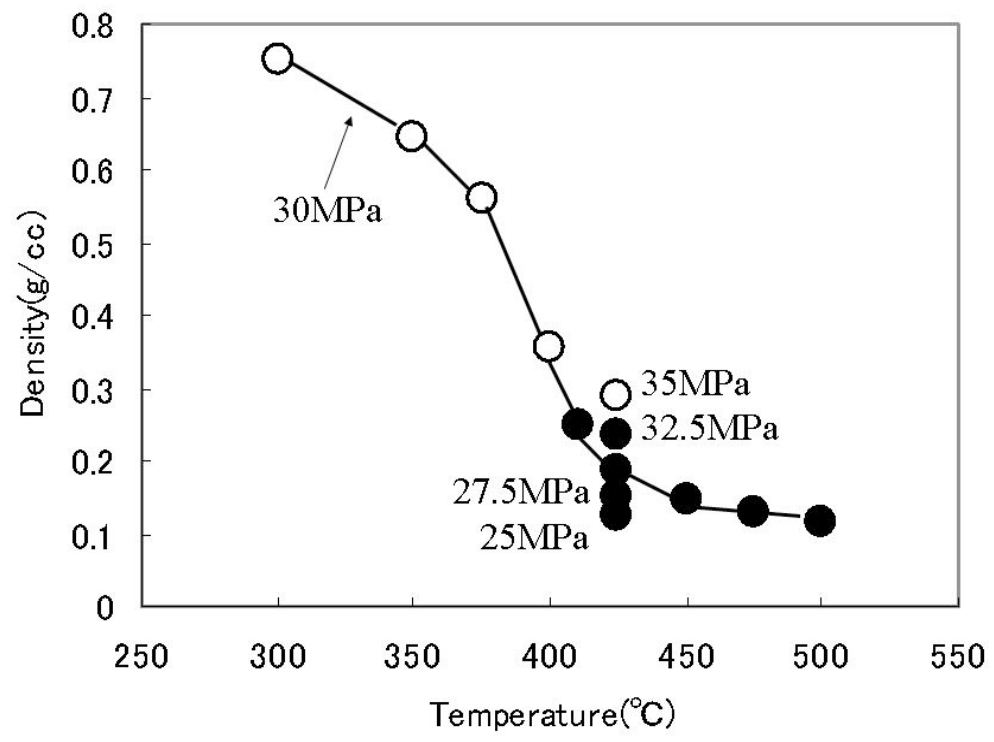

As another example, the effect of water density on polymorph of $\mathrm{BaTiO}_{3}$ particles synthesized hydrothermally under sub and supercritical water conditions have been examined. Aqueous $\mathrm{TiO}_{2}$ sols and $\mathrm{Ba}(\mathrm{OH})_{2}$ solution were used as starting materials. Hydrothermal synthesis was performed within the temperature range of 300 to $420{ }^{\circ} \mathrm{C}$, pressure of 20 to $40 \mathrm{MPa}$, and reaction time of 0.7 to $5 \mathrm{~s}$, where the density of water correspond from 0.15 to $0.7 \mathrm{~g} / \mathrm{cm}^{3}$. Figure 5 summarizes the relationship between water density and the crystal phase of $\mathrm{BaTiO}_{3}$. The crystal phase of products depends not only on the temperature but also the pressure. Under the subcritical conditions, cubic $\mathrm{BaTiO}_{3}$ was obtained, in which $\mathrm{OH}$ ions are substituted in the lattice oxygen, which is pseudo-cubic phase. The $\mathrm{BaTiO}_{3}$ particles with tetragonal phase were obtained under supercritical conditions, when the density of water was smaller than $0.5 \mathrm{~g} / \mathrm{cm}^{3}$. Dehydration might proceed at low density of water under supercritical conditions, thus, the residual $\mathrm{OH}$ ions in the lattice are reduced. Accordingly, tetragonal $\mathrm{BaTiO}_{3}$ can be obtained under supercritical conditions at density of water of $0.5 \mathrm{~g} / \mathrm{cm}^{3}$ or lower $[22,23]$. 
Figure 5. The relationship between $\mathrm{BaTiO}_{3}$ polymorph and hydrothermal reaction conditions. Open circle: cubic $\mathrm{BaTiO}_{3}$, Closed circle: tetragonal $\mathrm{BaTiO}_{3}$.

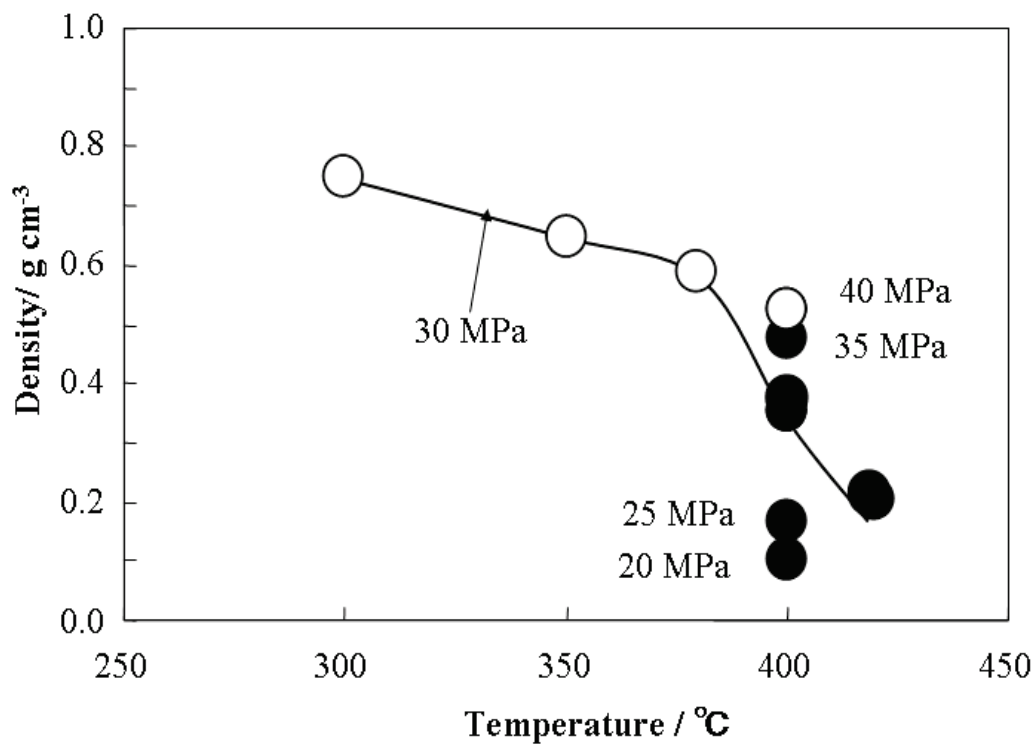

Short time hydrothermal reaction of $\mathrm{BaTiO}_{3}$ in supercritical water was conducted in order to reduce the particle size of $\mathrm{BaTiO}_{3}$. It is noteworthy that the reaction time affects the particle size and crystal phase of $\mathrm{BaTiO}_{3}$. Particle size of the $\mathrm{BaTiO}_{3}$ was as small as less than $10 \mathrm{~nm}$ with reducing the reaction time from $2 \mathrm{~s}$ to $7 \mathrm{~ms}$ (Figure 3c). However, Raman spectra and thermogravimetric analyses of $\mathrm{BaTiO}_{3}$ particles revealed that the product synthesized at $7 \mathrm{~ms}$ possess higher concentration of $\mathrm{OH}^{-}$ ions exhibited pseudo-cubic feature [24]. Cansell et al. showed that a flow reaction system for synthesizing $\mathrm{BaTiO}_{3}$ nanoparticles can be performed by hydrolyzing isopropoxide precursors in sub and supercritical water/ethanol mixture $\left(150-380{ }^{\circ} \mathrm{C}, 16 \mathrm{MPa}\right)$ [51]. The crystallinity of the as-prepared nanoparticles can easily be controlled by changing the water/ethanol ratio, since hydrolysis reaction occurs rapidly in systems with high water content, which is key for producing well-crystallized particles.

Applications for metal oxide nanoparticles are extending in many fields such as catalysts, electric devices, solid oxide fuel cell, magnetic storage, phosphor, optical materials, etc. One of the applicable forms for the inorganic nanoparticle is nanocomposite where the nanoparticles are homogeneously dispersed in polymer matrix. Refractive index is an important property for optical materials. Inorganic nanoparticles with high refractive indices have been dispersed in a variety of polymer matrices to obtain high refractive index nanocomposites [83-93]. Organic polymers have limited options for refractive indices compared with inorganic materials. The particle size of the dispersed phase is of critical importance to reduce the scattering loss. A dispersion of less than several tens of nanometers in size is necessary to obtain nanocomposites with high clarity. Titania and zirconia have high refractive indices. Highly transparent nanocomposites were successfully synthesized from a sulfonic acidmodified poly (bisphenol A carbonate) (PC) matrix and surface-modified $\mathrm{TiO}_{2}$ and $\mathrm{ZrO}_{2}$ nanoparticles. Surface modification of nanoparticles with phosphoric acid 2-ethylhexyl esters and introduction of sulfonic acid moiety into PC polymer matrix had a significant effect on the dispersion of nanoparticles. The refractive indices of the obtained nanocomposites increased with the amount of nanoparticles [27]. 
Flow reaction system is a simple system and continuous operation with short reaction (residence) time, all of which lead to more intensified materials formation processes. Thus, mixing between the precursor solution and supercritical water is a key to control the particle size and particle size distribution of product materials. In the flow reaction system, flow condition such as Reynold's number $[\mathrm{Re}]$ can be varied with flow rate and reactor tube inner diameter.

$$
[\mathrm{Re}]=\boldsymbol{r} \boldsymbol{v} / \mathrm{v}
$$

where $\boldsymbol{r}$ is tube inner diameter, $\boldsymbol{v}$ is the average flow rate and $v$ is kinetic viscosity which can be expressed as viscosity $\eta$ divided by density of fluid $\rho$.

Especially, since viscosity is markedly lowered under supercritical conditions, Re number tends to go up to turbulent flow region. The relations between average particle size of $\mathrm{BaTiO}_{3}$ or $\mathrm{Ti}$ conversion and flow condition (Re number) are shown in Figure 6. Average particle size of $\mathrm{BaTiO}_{3}$ tends to decrease as the finer reactor tube was used. Namely, average particle size of $\mathrm{BaTiO}_{3}$ decreased with an increase in Re number and converged to $35 \mathrm{~nm}$ as the Re number goes up to 10,000 where $\mathrm{Ti}$ conversion was attained to $100 \%$. Mixing is crucial for the particle size distribution and micro-mixer is the best way for rapid mixing of reactant solution and supercritical water. Thus, narrow particle size distribution can be achieved by the micro-mixing since hydrothermal reaction during heating period is prevented by good mixing at highly developed turbulent flow system.

Figure 6. Particle size and Ti conversion as a function of Re number.

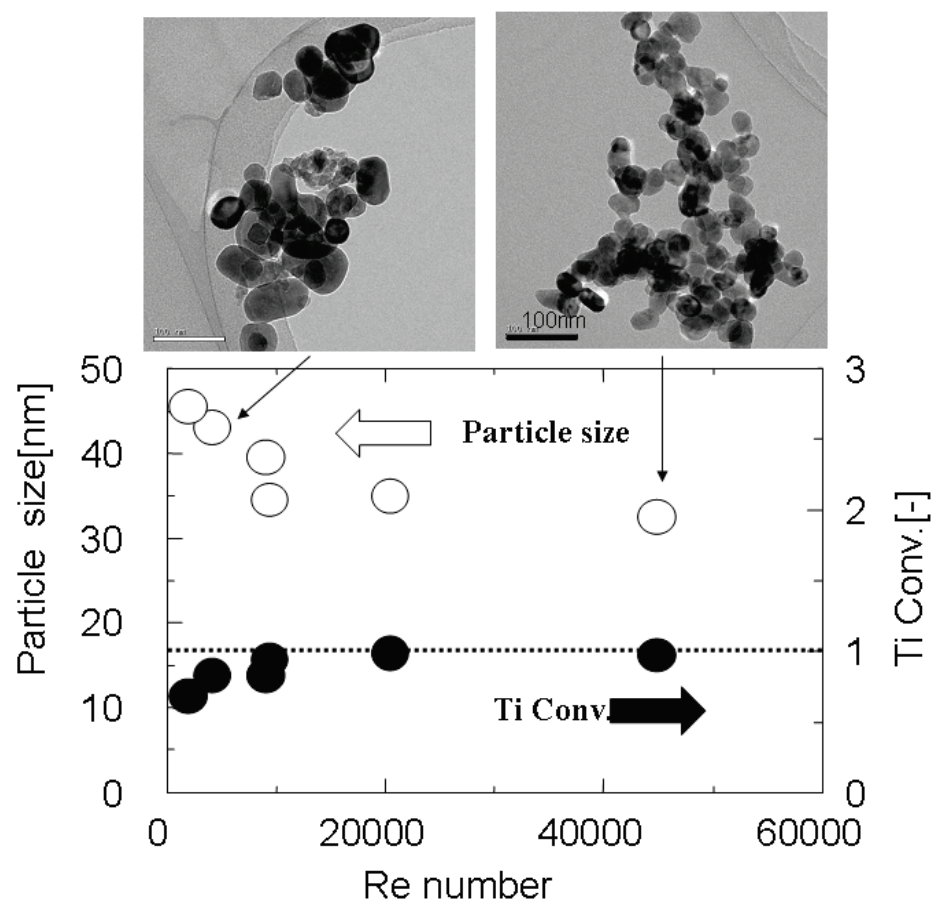

\subsection{Future Issues}

Since supercritical water can be obtained under high temperature and high pressure conditions, there is still limitations for in-situ measurements to elucidate the nanoparticles formation. Direct observation has been reported using diamond anvil cell (DAC) equipped with high speed camera and synchrotron radiation source for XRD measurements to elucidate the formation mechanism of $\mathrm{Zn}_{2} \mathrm{SiO}_{4}$ : $\mathrm{Mn}$ 
phosphor $[12,15]$. The DAC is a powerful tool for understanding nucleation and growth in hydrothermal synthesis of metal oxide particles in supercritical water. As already mentioned, $\alpha$-phase $\mathrm{Zn}_{2} \mathrm{SiO}_{4}: \mathrm{Mn}^{2+}$ crystallization in supercritical water was observed in-situ with DAC, and confirmed, by in situ XRD measurements under synchrotron radiation, that crystallization via homogeneous nucleation of $\alpha$-phase $\mathrm{Zn}_{2} \mathrm{SiO}_{4}$ requires only several tens of seconds. $\alpha$-phase $\mathrm{Zn}_{2} \mathrm{SiO}_{4}: \mathrm{Mn}^{2+}$ synthesized under supercritical conditions at $400{ }^{\circ} \mathrm{C}, 29 \mathrm{MPa}$ and reaction time of $90 \mathrm{~min}$ had an equivalent luminescence as that produced by the same raw materials with a firing process at $1200{ }^{\circ} \mathrm{C}$ for $240 \mathrm{~min}$.

Another powerful tool is estimation of solubility of inorganic species using chemical calculation on chemical species assuming equilibrium constants can be estimated as function of temperature, pressure and $\mathrm{pH}$ using a simplified HFK model. As already mentioned, solubility of metal oxides can be estimated using thermodynamic equilibria of dissociation constants, stabilities of complex formation, equations of mass balance and charge balance, ionic strength [28].

To realize the actual production of nanoparticles using the flow reaction system, there are still some problems in terms of engineering issues, namely, corrosion, blockage and mass production. Firstly, corrosion is an important issue for avoiding contamination of the product nanoparticles in flow reaction system. Corrosion takes place under the acidic or alkaline conditions. In the case of acidic region, pitting (localized corrosion) occurs in the presence of chloride ions under subcritical water conditions. Thus, cooling tubes are easily corroded and contamination from cooling tubes was remarkable and $\mathrm{Fe}$ and $\mathrm{Cr}$ are contaminated in the product powders. To solve the problem, highcorrosion resistant materials such as $\mathrm{Ti}$ and $\mathrm{Ta}$ are used as inner side of composite reaction tube where outer tube was made of metal alloys such as stainless and $\mathrm{Ni}$ alloys with high mechanical strength under high temperature and high pressure conditions. Accordingly, reactor material and configuration is a strong consideration to prevent contamination through corrosion and reliable operation to produce fine particles without impurity.

Secondly, the issue of blockage is the most troublesome problem. Blockage can take place at the mixing tee, reactor tube and cooling tube and back pressure regulator. This terrible blockage is caused by nanofibers, nanorods such as potassium hexatitanates, where nanorods are aggregated and blocked and accumulate inside the mixing tee. Another blockage takes place in the reactor and cooling tubes where nanoparticles attached to the inner side of the tube and accumulate to thick layers until finally the tube is filled with nanoparticles. High flow rate is the solution to avoid blockages, because turbulent flow stops accumulation of particles in the tube reactor. In addition, density of water is an important factor to avoid particle sedimentation in the reactor tube. At low density of water, nanoparticles easily settle down and residence time might become longer and large particles form due to crystal growth.

The last issue, mass production, is of importance to realize the industrialization for nanoparticle production. As a scale-up strategy, a pilot-scale supercritical hydrothermal synthesis unit with a capacity of 30 ton/year was constructed and is running in Korea [94]. The strategy for mass production by numbering-up, and operation at high concentrations has yet to be realized. As a mixing device, union tee mixer is usually used to combine two streams of the starting materials solution and supercritical water. The numbering-up system is better for mixing rather than the scale-up system, 
where mixing between starting solution and supercritical water is not rapid enough to give wide particle size distribution. Hydrodynamics around the mixing point of the starting solution and supercritical water is one of the most important factors for particle formation from the view point of material science and processing. A micro-reaction system for high temperature and high pressure water has been under-consulted in our research institute and numbering-up system of mixing parts will be proposed in the near future.

\section{Experimental Section}

\subsection{Supercritical Hydrothermal Batchwise System}

Batchwise hydrothermal synthesis was carried out using an autoclave type reactor made from Inconel 625 (Ni-Cr alloys), with inner volume $\left(500 \mathrm{~cm}^{3}\right)$ and operation maximum temperature and pressure of $500{ }^{\circ} \mathrm{C}$ and $100 \mathrm{MPa}$. The mixture of starting materials was prepared as aqueous slurry. The slurry was put into a gold-tube reactor inside the autoclave. Here, the corrosion-resistant and heatstable gold-tube reactor was necessary due to the relatively high reaction temperature in comparison with the conventional hydrothermal method. Hydrothermal synthesis was carried out at $400{ }^{\circ} \mathrm{C}$ with autogenous pressure of $25 \mathrm{MPa}$.

\subsection{Supercritical Hydrothermal Flow System}

Schematic diagram of the flow reactor system used in this study is shown in Figure 7. Each of precursor solution and $\mathrm{KOH}$ solution were fed to a reactor by high-pressure pump at a flow rate of $8 \mathrm{~g} / \mathrm{min}$, and these two streams were mixed at the first mixing point, MP1. On the other hand, distilled water was fed by another high-pressure pump at a flow rate of $44 \mathrm{~g} / \mathrm{min}$ and heated to an appropriate temperature by an electric furnace. The reactant mixture of precursors and $\mathrm{KOH}$ was mixed with the supercritical water at the second mixing point MP2. The residence time from MP1 to MP2 was varied with reactor tube volume determined by the inner diameter and length of the reactor tube.

Reaction (Residence) time, $\boldsymbol{t}$, was calculated using Equation (5):

$$
\boldsymbol{t}=\boldsymbol{V} / \boldsymbol{F}\left(\rho_{298} / \rho_{\mathrm{T}}\right)
$$

where $\boldsymbol{F}$ is the total flow rate $(\mathrm{g} / \mathrm{s})$ and $\boldsymbol{V}$ is the reactor volume $\left(\mathrm{cm}^{3}\right) \rho_{298}$ and $\rho_{\mathrm{T}}$ are densities of pure water $\left(\mathrm{g} / \mathrm{cm}^{3}\right)$ at $298 \mathrm{~K}$ and reaction temperature, respectively.

For an example, in one case, the temperature and pressure of the hydrothermal reaction were set to be $400{ }^{\circ} \mathrm{C}$ and $30 \mathrm{MPa}$, respectively. The reactant was maintained at $400{ }^{\circ} \mathrm{C}$ for $8 \mathrm{~ms}$ in a $1 / 16$ inch SUS tube reactor (inner diameter: $0.8 \mathrm{~mm}$ and length: $14 \mathrm{~cm}$ ). After the prescribed reaction time period, the hydrothermal reaction was quenched by cooling at the end of the reactor. The reactant solution was depressurized with a back pressure regulator.

Particles were recovered as a slurry solution, separated with a membrane filter (pore size $200 \mathrm{~nm}$ ) as aggregation, washed with pure water (and diluted acetic acid in case of $\mathrm{BaTiO}_{3}$ ), and then dried at $60{ }^{\circ} \mathrm{C}$ in an electric oven for 24 hours. The crystal structure of the products was determined by X ray diffraction measurement (XRD; Rigaku Co. Ltd., Model RINT 2000). Particle size and the morphology 
of the obtained particles were examined by scanning electron microscopy (SEM; JEOL Co. Ltd., Model JSM-5600) or by transmission electron microscopy (TEM; FEI Co., Model TECNAI-G2).

Figure 7. Schematic diagram of the flow reactor system: (1) feedstock of starting materials solution; (2) feedstock of $\mathrm{KOH}$ solution; (3) feedstock of distilled water; (4-6) highpressure pump; $(7,8)$ electric furnace; $(9)$ heat exchanger; $(10)$ backpressure regulator; $(11)$ filtrate reservoir.

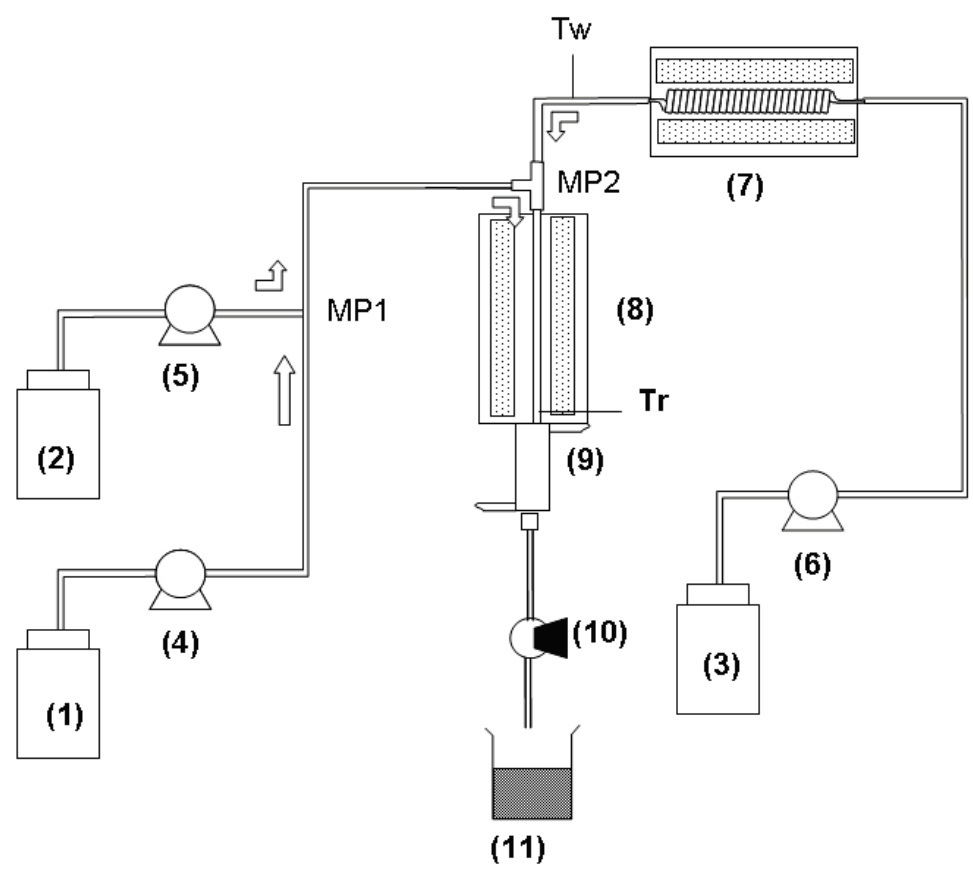

\section{Conclusions}

This review paper describes advantages of hydrothermal synthesis for particle formation using supercritical water and a brief survey of our research. An outstanding feature of supercritical water as a particle formation medium is to control the crystal phase through adjustable solvent properties such as density of water. In addition, particle formation using supercritical water can reduce the alkaline concentration and keep it free of toxic organic solvents, thus, the hydrothermal process is compatible with green and sustainable chemistry in a way that reduces the environment load. We expect that transfer of supercritical hydrothermal technology of material processing from laboratory to industry will be achieved in the near future in combining economical efficiency and sustainable development.

\section{References}

1. Adschiri, T.; Hakuta, Y.; Arai, K. Hydrothermal synthesis of metal oxide fine particles at supercritical conditions. Ind. Eng. Chem. Res. 2000, 39, 4901-4907.

2. Adschiri, T.; Hakuta, Y.; Sue, K.; Arai, K. Hydrothermal synthesis of metal oxide nanoparticles at supercritical conditions. J. Nanopart. Res. 2000, 3, 227-235.

3. Hakuta, Y.; Hayashi, H.; Arai, K. Fine particle formation using supercritical fluids. Curr. Opin. Solid State Mat. Sci. 2003, 7, 341-351. 
4. Hayashi, H.; Torii, K. Hydrothermal synthesis of titania photocatalyst under subcritical and supercritical water conditions. J. Mater. Chem. 2002, 12, 3671-3676.

5. Yahya, R.B.; Hayashi, H.; Nagase, T.; Ebina, T.; Onodera, Y.; Saitoh, N. Hydrothermal synthesis of potassium hexatitanates under subcritical and supercritical water conditions and its application in photocatalysts. Chem. Mater. 2001, 13, 842-847.

6. Hayashi, H.; Hakuta, Y.; Kurata, Y. Hydrothermal synthesis of potassium niobate photocatalysts under subcritical and supercritical water conditions. J. Mater. Chem. 2004, 14, 2046-2051.

7. Li, B.; Hakuta, Y.; Hayashi, H. Hydrothermal synthesis of $\mathrm{KNbO}_{3}$ powders in supercritical water and its nonlinear optical properties. J. Supercrit. Fluid. 2005, 35, 254-259.

8. Li, B.; Hakuta, Y.; Hayashi, H. Hydrothermal synthesis of crystalline rectangular titanoniobate particles. Chem. Commum. 2005, 1732-1734.

9. Li, B.; Hakuta, Y.; Hayashi, H. Synthesis of potassium titanoniobate in supercritical and subcritical water and investigations on its photocatalytic performance. J. Supercrit. Fluid. 2006, $39,63-69$.

10. Li, B.; Hakuta, Y.; Hayashi, H. The synthesis of titanoniobate compound characteristic of various particle morphologies through a novel solvothermal route. Mater. Lett. 2007, 61, 3791-3794.

11. Hayashi, H.; Hakuta, Y. Hydrothermal epitaxy of $\mathrm{KTaO}_{3}$ thin films under supercritical water conditions. J. Mater. Sci. 2008, 43, 2342-2347.

12. Takesue, M.; Shimoyama, K.; Murakami, S.; Hakuta, Y.; Hayashi, H.; Smith, R.L., Jr. Phase formation of Mn-doped zinc silicate in water at high-temperatures and high-pressures. $J$. Supercrit. Fluid. 2007, 43, 214-221.

13. Takesue, M.; Suino, A.; Hakuta, Y.; Hayashi, H.; Smith, R.L., Jr. Formation mechanism and luminescence appearance of Mn-doped zinc silicate particles synthesized in supercritical water. $J$. Solid State Chem. 2008, 181, 1307-1313.

14. Takesue, M.; Suino, A.; Shimoyama, K.; Hakuta, Y.; Hayashi, H.; Smith, R.L., Jr. Formation of $\alpha$ and $\beta$-phase Mn-doped zinc silicate in supercritical water and its luminescent properties at $\mathrm{Si} /(\mathrm{Zn}+\mathrm{Mn})$ ratios from 0.25 to 1.25 . J. Cryst. Growth 2008, 310, 4185-4189.

15. Takesue, M.; Shimoyama, K.; Shibuki, K.; Suino, A.; Hakuta, Y.; Hayashi, H.; Ohishi, Y.; Smith, R.L., Jr. Formation of Mn-doped zinc silicate in supercritical water followed with in situ synchrotron radiation X-ray diffraction. J. Supercrit. Fluid. 2009, 49, 351-355.

16. Takesue, M.; Suino, A.; Hakuta, Y.; Hayashi, H.; Smith, R.L., Jr. Crystallization trigger of Mndoped zinc silicate in supercritical water via $\mathrm{Zn}, \mathrm{Mn}, \mathrm{Si}$ sources and complex agent ethylenediamine tetraacetic acid. Mater. Chem. Phys. 2010, 121, 330-334.

17. Hakuta, Y.; Shimoyachi, K.; Hayashi, H.; Arai, K. Hydrothermal synthesis of potassium hexatitanate photocatalyst under supercritical water conditions. J. Ion Exch. 2003, 14, 393-396.

18. Hakuta, Y.; Hayashi, H.; Arai, K. Hydrothermal synthesis of photocatalyst potassium hexatitanate nanowires under supercritical water conditions. J. Mater. Sci. 2004, 39, 4977-4980.

19. Hakuta, Y.; Ohashi, T.; Hayashi, H.; Arai, K. Hydrothermal synthesis of zirconia nanocrystals in supercritical water. J. Mater. Res. 2004, 19, 2230-2234. 
20. Hakuta, Y.; Ura, H.; Hayashi, H.; Arai, K. Effects of hydrothermal synthetic conditions on the particle size of $\gamma$ - $\square \mathrm{AlO}(\mathrm{OH})$ in sub and supercritical water using a flow reaction system. Mater. Chem. Phys. 2005, 93, 466-472.

21. Noguchi, T.; Matsui, K.; Islam, N.M.; Hakuta, Y.; Hayashi, H. Rapid synthesis of $\gamma-\mathrm{Al}_{2} \mathrm{O}_{3}$ nanoparticles in supercritical water by continuous hydrothermal flow reaction system. J. Supercrit. Fluid. 2008, 46, 129-136.

22. Hakuta, Y.; Ura, H.; Hayashi, H.; Arai, K. Effect of water density on polymorph of $\mathrm{BaTiO}_{3}$ nanoparticles synthesized under sub and supercritical water conditions. Mater. Lett. 2005, 59, 1387-1390.

23. Hakuta, Y.; Ura, H.; Hayashi, H.; Arai, K. Continuous production of $\mathrm{BaTiO}_{3}$ nanoparticles by hydrothermal synthesis. Ind. Eng. Chem. Res. 2005, 44, 840-846.

24. Matsui, K.; Noguchi, T.; Islam, N.M.; Hakuta, Y.; Hayashi, H. Rapid synthesis of $\mathrm{BaTiO}_{3}$ nanoparticles in supercritical water by continuous hydrothermal flow reaction system. J. Cryst. Growth 2008, 310, 2584-2589.

25. Lu, J.; Hakuta, Y.; Hayashi, H.; Ohashi, T.; Nagase, T.; Hoshi, Y.; Sato, K.; Nishioka, M.; Inoue T.; Hamakawa, S. Preparation of $\mathrm{Ca}_{0.8} \mathrm{Sr}_{0.2} \mathrm{Ti}_{1-\mathrm{x}} \mathrm{Fe}_{\mathrm{x}} \mathrm{O}_{3-}(\mathrm{x}=0.1-0.3)$ nanoparticles using a flow supercritical reaction system. J. Supercrit. Fluid. 2008, 46, 77-82.

26. Hayashi, H.; Ueda, A.; Suino, A.; Hiro, K.; Hakuta, Y. Hydrothermal synthesis of yttria stabilized $\mathrm{ZrO}_{2}$ nanoparticles in subcritical and supercritical water using a flow reaction system. J. Solid State Sci. 2009, 182, 2985-2990.

27. Imai, Y.; Terahara, A.; Hakuta, Y.; Matsui, K.; Hayashi, H.; Ueno, N. Transparent poly(bisphenol A carbonate)-based nanocomposites with high refractive index nanoparticles. Euro. Poly. J. 2009, 45, 630-638.

28. Sue, K.; Suzuki, M.; Arai, K.; Ohashi, T.; Ura, H.; Matsui, K.; Hakuta, Y.; Hayashi, H.; Watanabe, M.; Hiaki, T. Size-controlled synthesis of metal oxide nanoparticles with a flowthrough supercritical water method. Green Chem. 2006, 8, 634-638.

29. Goh, G.K.L.; Lange, F.F.; Haile, S.M.; Levi, C.G. Hydrothermal syntheis of $\mathrm{KNbO}_{3}$ and $\mathrm{NaNbO}_{3}$ powders. J. Mater. Res. 2003, 18, 338-345.

30. Lu, C.-H.; Lo, S.-Y.; Wang, Y.-L. Glycothermal preparation of potassium niobate ceramic particles under supercritical conditions. Mater. Lett. 2002, 55, 121-125.

31. Goh, G.K.L.; Haile, S.M.; Levi, C.G.; Lange, F.F. Hydrothermal synthesis of perovskite and pyrochlore powders of potassium tantalate. J. Mater. Res. 2002, 17, 3168-3174.

32. Sorescua, M.; Diamandescua, L.; Tarabasanu, D. $\alpha-\mathrm{Fe}_{2} \mathrm{O}_{3}-\mathrm{In}_{2} \mathrm{O}_{3}$ mixed oxide nanoparticles synthesized under hydrothermal supercritical conditions. J. Phys. Chem. Solids 2004, 65, 1719-1725.

33. Lee, J.; Teja, A.S. Characteristics of lithium iron phosphate $\left(\mathrm{LiFePO}_{4}\right)$ particles synthesized in subcritical and supercritical water. J. Supercrit. Fluid. 2005, 35, 83-90.

34. Umetsu, M.; Man, X.; Okuda, K.; Tahereh, M.; Ohara, S.; Zhang, J.; Takami, S.; Adschiri, T. Biomass-assisted hydrothermal synthesis of ceria nanoparticle-A new application of lignin as a bio-nanopool. Chem. Lett. 2006, 35, 732-733. 
35. Zheng, Q.X.; Li, B.; Xue, M.; Zhang, H.D.; Zhan, Y.J.; Pang, W.S.; Tao, X.T.; Jiang, M.H. Synthesis of $\mathrm{YVO}_{4}$ and rare earth-doped $\mathrm{YVO}_{4}$ ultra-fine particles in supercritical water. $J$. Supercrit. Fluid. 2006, 39, 63-69.

36. Zhao, D.; Han, E.; Wu, X.; Guan, H. Hydrothermal synthesis of ceria nanoparticles supported on carbon nanotubes in supercritical water. Mater. Lett. 2006, 60, 3544-3547.

37. Xu, C.B.; Teja, A.S. Supercritical water synthesis and deposition of iron oxide $\left(\right.$ alpha- $\left.\mathrm{Fe}_{2} \mathrm{O}_{3}\right)$ nanoparticles in activated carbon. J. Supercrit. Fluid. 2006, 39, 135-141.

38. Yoon, M.J.; In, J.H.; Lee, H.C.; Lee, C.H. Comparison of YAG:Eu phosphor synthesized by supercritical water and solid-state methods in a batch reactor. Korean J. Chem. Eng. 2006, 23, 842-846.

39. Zhao, D.; Wu, X.; Guan, H.; Han, E. Study on supercritical hydrothermal synthesis of $\mathrm{CoFe}_{2} \mathrm{O}_{4}$ nanoparticles. J. Supercrit. Fluid. 2007, 42, 226-233.

40. Assaaoudi, H.; Fang, Z.; Butler, I.S.; Ryan, D.H.; Kozinski, J.A. Characterization of a new magnesium hydrogen orthophosphate salt, $\mathrm{Mg}_{3.5} \mathrm{H}_{2}\left(\mathrm{PO}_{4}\right)_{3}$, synthesized in supercritical water. Solid State Sci. 2007, 9, 385-393.

41. Assaaoudi, H.; Fang, Z.; Butler, I.S.; Kozinski, J.A. Synthesis of erbium hydroxide microflowers and nanostructures in subcritical water. Nanotechnology 2008, 19, 185606.

42. Sato, T.; Sue, K.; Tsumatori, H.; Suzuki, M.; Tanaka, S.; Kawai-Nakamura, A.; Saitoh, K.; Aida, K.; Hiaki, T. Hydrothermal synthesis of $\mathrm{CuAlO}_{2}$ with the delafossite structure in supercritical water. J. Supercrit. Fluid. 2008, 46, 173-177.

43. Viswanathan, R.; Gupta, R.B. Formation of zinc oxide nanoparticles in supercritical water. $J$. Supercrit. Fluid. 2003, 27, 187-193.

44. Hao, Y.; Teja, A.S. Continuous hydrothermal crystallization of $\alpha-\mathrm{Fe}_{2} \mathrm{O}_{3}$ and $\mathrm{Co}_{3} \mathrm{O}_{4}$ nanoparticles. J. Mater. Res. 2003, 18, 415-422.

45. Cote, L.J.; Teja, A.S.; Wilkinson, A.P.; Zhang, Z.J. Continuous hydrothermal synthesis of $\mathrm{CoFe}_{2} \mathrm{O}_{4}$ nanoparticles. Fluid Phase Equilibria 2003, 210, 307-317.

46. Hakuta, Y.; Haganuma, T.; Sue, K.; Adschiri, T.; Arai, K. Continuous production of phosphor YAG:Tb nanoparticles by hydrothermal synthesis in supercritical water. Mater. Res. Bull. 2003, $38,1257-1265$.

47. Ohara, S.; Mousavand, T.; Umetsu, M.; Takami, S.; Adschiri, T.; Kuroki, Y.; Takata, M. Hydrothermal synthesis of fine zinc oxide particles under supercriticalconditions. Solid State Ionics 2004, 172, 261-264.

48. Sue, K.; Kimura, K.; Murata, K.; Arai, K. Effect of cations and anions on properties of zinc oxide particles synthesized in supercritical water. J. Supercrit. Fluid. 2004, 30, 325-331.

49. Sue, K.; Kimura, K.; Yamamoto, M.; Arai, K. Rapid hydrothermalsynthesis of ZnO nanorods without organics. Mater. Lett. 2004, 58, 3350-3352.

50. Lee, H.-C.; Kim, J.-J.; In, J.-H.; Lee, C.-H. NaFeEDTA decomposition and hematite nanoparticle formation in supercritical water oxidation. Ind. Eng. Chem. Res. 2005, 44, 6615-6621.

51. Reverón, H.; C. Aymonier, H.; Loppinet-Serani, A.; Elissalde, C.; Maglione, M.; Cansell, F. Single-step synthesis of well-crystallized and pure barium titanate nanoparticles in supercritical fluids. Nanotechnology 2005, 16, 1137-1143. 
52. Elissalde, C.; Reverón, H.; Aymonier, C.; Michau, D.; Cansell, F.; Maglione, M. The ferroelectric transition temperature as an intrinsic probe for sinterednanocrystalline $\mathrm{BaTiO}_{3}$ synthesized under supercritical conditions. Nanotechnology 2005, 16, 797-802.

53. Reverón, H.; Elissalde, C.; Aymonier, C.; Bidault, O.; Maglione, M.; Cansell, F. Supercritical fluid route for synthesizing crystalline barium strontium titanate nanoparticles. J. Nanosci. Nanotechnol. 2005, 5, 1741-1745.

54. Reverón, H.; Elissade, C.; Aymonier, C.; Bousquet, C.; Maglione, M.; Cansell, F. Continuous supercritical synthesis and dielectric behavior of the whole BST solid solution. Nanotechnology 2006, 17, 3527-3532.

55. Lee, J.H.; Ham, J.Y. Synthesis of manganese oxide particles in supercritical water. Korean J. Chem. Eng. 2006, 23, 714-719.

56. Levy, C.; Watanabe, M.; Aizawa, Y.; Inomata, H.; Sue, K. Synthesis of nanophased metal oxides in supercritical water. Catalysts for biomass conversion. Int. J. Appl. Ceram. Technol. 2006, 3, 337-344.

57. Fang, Z.; Assaaoudi, H.; Guthrie, R.I.L.; Kozinski, J.A. Continuous synthesis of tin and indium oxide nanoparticles in sub- and supercritical water. J. Amer. Ceram. Soc. 2007, 90, 2367-2371.

58. Cabanas, A.; Li, J.; Blood, P.; Chudoba, T.; Lojkowski, W.; Poliakoff, M.; Lester, E. Synthesis of nanoparticulate yttrium aluminum garnet in supercritical water-ethanol mixtures. J. Supercrit. Fluid. 2007, 40, 284-292.

59. In, J.H.; Lee, H.C.; Yoon, M.J.; Lee, K.K.; Lee, J.W.; Lee, C.H. Syntesis of nano-sized YAG: $\mathrm{Eu}^{3+}$ phosphor in continuous supercritical water system. J. Supercrit. Fluid. 2007, 40, 389-396.

60. Weng, X.L.; Boldrin, P.; Abrahams, I.; Skinner, S.J.; Darr, J.A. Direct syntheses of mixed ion and electronic conductors $\mathrm{La}_{4} \mathrm{Ni}_{3} \mathrm{O}_{10}$ and $3 \mathrm{Ni}_{2} \mathrm{O}_{7}$ from nanosized coprecipitates. Chem. Mater. 2007, $19,4382-4384$.

61. Yoon, M.J.; Bae, Y.S.; Son, S.H.; Lee, J.W.; Lee, C.H. Comparison of YAG: Eu phosphors synthesized by supercritical water in batch and continuous reactors. Korean J. Chem. Eng. 2007, $24,877-880$.

62. Zhang, J.; Ohara, S.; Umetsu, M.; Naka, T.; Hatakeyama, Y.; Adschiri, T. Colloidal ceria nanocrystals: A tailor-made crystal morphology in supercritical water. Adv. Mater. 2007, 19, 203-206.

63. Rangappa, D.; Ohara, S.; Naka, T.; Kondo, A.; Ishii, M.; Adschiri, T. Synthesis and organic modification of $\mathrm{CoAl}_{2} \mathrm{O}_{4}$ nanocrystals under supercritical water conditions. J. Mater. Chem. 2007, 17, 4426-4429.

64. Rangappa, D.; Ohara, S.; Naka, T.; Kondo, A.; Ishii, M.; Kobayashi, T.; Adschiri, T. Transparent $\mathrm{CoAl}_{2} \mathrm{O}_{4}$ hybrid nano pigment by organic ligand-assisted supercritical water. J. Amer. Chem. Soc. 2007, 129, 11061-11066.

65. Mousavand, T.; Zhang, J.; Ohara, S.; Umetsu, M.; Naka, T.; Adschiri, T. Organic ligand-assisted supercritical hydrothermal synthesis of titanium oxide nano-crystals leading to perfect dispersed titanium oxide nanoparticle in organic phase. J. Nanopart. Res. 2007, 9, 1067-1071. 
66. Mousavand, T.; Ohara, S.; Umetsu, M.; Zhang, J.; Takami, S.; Naka, T.; Adschiri, T. Hydrothermal synthesis and in situ surface modification of boemite nano-particles in supercritical water. J. Supercrit. Fluid. 2007, 40, 397-401.

67. Becker, J.; Hald, P.; Bremholm, M.; Pedersen, J.S.; Chevallier, J.; Iversen, S.B. ; Iversen, B.B. Critical size of crystalline $\mathrm{ZrO}_{2}$ nanoparticles synthesized in near- and supercritical water and supercritical isopropyl alcohol. ACS Nano 2008, 2, 1058-1068.

68. Fang, Z.; Assaaoudi, H.; Lin, H.B.; Wang, X.M.; Butler, I.S.; Kozinski, J.A. Synthesis of nanocrystalline $\mathrm{SnO}_{2}$ in supercritical water. J. Nanopart. Res. 2007, 9, 683-687.

69. Sato, T.; Sue, K.; Akiyama, Y.; Shibata, K.; Kawasaki, S.I.; Tanaka, S.; Saitoh, K.; KawaiNakamura, A.; Aida, K.; Hiaki, T. Effect of $\mathrm{pH}$ on hydrothermal synthesis of gamma- $\mathrm{Al}_{2} \mathrm{O}_{3}$ nanoparticles at $673 \mathrm{~K}$. Chem. Lett. 2008, 37, 242-243.

70. Xu, C.B.; Teja, A.S. Continuous hydrothermal synthesis of iron oxide and PVA-protected iron oxide nanoparticles. J. Supercrit. Fluid. 2008, 44, 85-91.

71. Xu, C.B.; Lee, J.; Teja, A.S. Continuous hydrothermal synthesis of lithium iron phosphate particles in subcritical and supercritical water. J. Supercrit. Fluid. 2008, 44, 92-97.

72. Atashfaraz, M.; Shariaty-Niassar, M.; Ohara, S.; Minami, K.; Umetsu, M.; Naka, T.; Adschiri, T. Effect of titanium dioxide solubility on the formation of $\mathrm{BaTiO}_{3}$ nanoparticles in supercritical water. Fluid Phase Equilibria 2007, 257, 233-237.

73. Aimable, A.; Xin, B.; Millot, N.; Aymes, D. Continuous hydrothermal synthesis of nanometric $\mathrm{BaZrO}_{3}$ in supercritical water. J. Solid State Chem. 2008, 181, 183-189.

74. Sato, T.; Sue, K.; Suzuki, W.; Suzuki, M.; Matsui, K.; Hakuta, Y.; Hayashi, H.; Arai, K.; Kawasaki, S. I.; Kawai-Nakamura, A.; Hiaki, T. Rapid and continuous production of ferrite nanoparticles by hydrothermal synthesis at $673 \mathrm{~K}$ and $30 \mathrm{MPa}$. Ind. Eng. Chem. Res. 2008, 47, $1855-1860$.

75. Chaudhry, A.A.; Goodall, J.; Vickers, M.; Cockcroft, J.K.; Rehman, I.; Knowles, J.C.; Darr, J.A. Synthesis and characterization of magnesium substituted calcium phosphate bioceramic nanoparticles made via continuous hydrothermal flow synthesis. J. Mater. Chem. 2008, 18, 5900-5908.

76. Weng, X.L.; Boldrin, P.; Abrahams, I.; Skinner, S.J.; kellici, S.; Darr, J.A. Direct syntheses of $\mathrm{La}_{\mathrm{n}+1} \mathrm{Ni}_{\mathrm{n}} \mathrm{O}_{3 \mathrm{n}+1}$ phases $(\mathrm{n}=1,2,3$ and infinity) from nanosized cocrystallites. J. Solid State Chem. 2008, 181, 1123-1132.

77. Kim, J.R.; Myeong, W.J.; Ihm, S.K. Characteristics in oxygen strage capacity of ceria-zirconia mixed oxides prepared by continuous hydrothermal synthesis in supercritical water. Appl. Catal. B-Environ. 2007, 71, 57-63.

78. Boldrin, P.; Hebb, A.K.; Chaudhry, A.A.; Otley, L.; Thiebaut, B.; Bishop, P.; Darr, J.A. Direct syntheses of nanosized $\mathrm{NiCo}_{2} \mathrm{O}_{4}$ spinel and related compounds via continuous hydrothermal synthesis methods. Ind. Eng. Chem. Res. 2007, 46, 4830-4838.

79. Ohara, S.; Mousavand, T.; Sasaki, T.; Umetsu, M.; Naka, T.; Adschiri, T. Continuous production of fine zinc oxide nanorods by hydrothermal synthesis in supercritical water. J. Mater. Sci. 2008, 43, 2393-2396. 
80. Takami, S.; Ohara, S.; Adschiri, T.; Wakayama, Y.; Chikyow, T. Continuous synthesis of organicinorganic hybridized cubic nanoassemblies of octahedral cerium oxide nanocrystals and hexanedioic acid. Dalton Trans. 2008, 40, 5442-5446.

81. Kim, J.; Park, Y.S.; Veriansyah, B.; Kim, J.D.; Lee, Y.W. Continuous synthesis of surfacemodified metal oxide nanoparticles using supercritical methanol for highly stabilized nanofluids. Chem. Mater. 2008, 20, 6301-6303.

82. Chudoba, T.; Lester, E.; Lojkowski, W.; Poliakoff, M.; Li, J.; Grzanka, E.; Presz, A. Synthesis of nano-sized yttrium-aluminum garnet in a continuous-flow reactor in supercritical fluids. $Z$. Naturforsch. Sect. B 2008, 63, 756-764.

83. Wang, B.; Wilkers, G.L. New Ti-PTMO and Zr-PTMO creamer hybrid materials prepared by the sol gel method: Synthesis and characterization. J. Polym. Sci. Part A-Polym. Chem. 1991, 29, 905-909.

84. Wang, B.; Wilkers, G.L.; Smith, C.D.; McGrath, J.E. High refractive index hybrid creamer materials prepared from titanium tetraisopropoxide and poly(arylene ether phosphine oxide) through sol-gel processing. Polym. Commun. 1991, 32, 400-402.

85. Wang, B.; Wilkers, G.L.; Hendrick, J.C.; Liptak, S.C.; McGrath, J.E. New high refractive index organic/inorganic hybrid materials from sol-gel processing. Macromolecules 1991, 24, 3449-3450.

86. Nussbaumer, R.J.; Caseri, W.R.; Smith, P.; Tervoort, T. Polymer-TiO ${ }_{2}$ nanocomposites: A route towards visually transparent broadband UV filters and high refractive index materials. Macromol. Mater. Eng. 2003, 288, 44-49.

87. Lu, C.; Cui, Z.; Guan, J.; Yang, B.; Shen, J. Research on preparation, structure and properties of $\mathrm{TiO}_{2}$ /polythiourethane hybrid optical films with high refractive index. Macromol. Mater. Eng. 2003, 288, 717-723.

88. Xiong, M.; Zhou, S.; Wu, L.; Wang, B.; Yang, L. Sol-gel derived organic-inorganic hybrid from trialkoxysilane-capped acrylic resin and titania: effects of preparation conditions on the structure and properties. Polymer 2004, 45, 8127-8138.

89. Chen, W.C.; Liu, W.C.; Wu, P.T.; Chen, P.F. Synthesis and characterization of oligomeric phenylsilsesquioxane-titania hybrid optical films. Mater. Chem. Phys. 2004, 83, 71-77.

90. Nakayama, N.; Hayashi, T. Preparation and characterization of $\mathrm{TiO}_{2}-\mathrm{ZrO}_{2}$ and thiol-acrylate resin nanocompostes with high refractive indez via UV-induced crosslinking polymerization. Compos. Part A-Appl. Sci. Manuf. 2007, 38, 1996-2004.

91. Liu, J.G.; Nakamura, Y.; Ogura, T.; Shibasaki, Y.; Ando, S.; Ueda, M. Optically transparent sulfur-containing polyimide- $\mathrm{TiO}_{2}$ nanocomposite films with high refractive index and negative pattern formation from poly(amic acid) $-\mathrm{TiO}_{2}$ nanocomposite film. Chem. Mater. 2008, 20, $273-281$.

92. Su, H.W.; Chen, W.C. High refractive index polyimide-nanocrystalite-titania hybrid optical materials. J. Mater. Chem. 2008, 18, 1139-1145.

93. Lee, S.; Shin, H.J.; Yoon, S.M.; Yi, D.K.; Choi, J.Y.; Paik, U. Refractive index engineering of transparent $\mathrm{ZrO}_{2}$-polydimethylsiloxane nanocomposites. J. Mater. Chem. 2008, 18, 1751-1755. 
94. Lee, Y.-W. Formation of nano particles in supercritical fluids. In Proceedings of ISHR \& ICSTR, Sendai, Japan, 5-9 August 2006.

(C) 2010 by the authors; licensee MDPI, Basel, Switzerland. This article is an Open Access article distributed under the terms and conditions of the Creative Commons Attribution license (http://creativecommons.org/licenses/by/3.0/). 